\title{
Searching for illusory motion
}

\author{
Ian M. Thornton ${ }^{1}$ • Sunčica Zdravković ${ }^{2,3}$
}

Published online: 22 May 2019

(C) The Psychonomic Society, Inc. 2019

\begin{abstract}
In a series of four experiments, standard visual search was used to explore whether the onset of illusory motion pre-attentively guides vision in the same way that the onset of real-motion is known to do. Participants searched for target stimuli based on Akiyoshi Kitaoka's classic illusions, configured so that they either did or did not give the subjective impression of illusory motion. Distractor items always contained the same elements as target items, but did not convey a sense of illusory motion. When target items contained illusory motion, they popped-out, with flat search slopes that were independent of set size. Search for control items without illusory motion - but with identical structural differences to distractors - was slow and serial in nature ( $>200 \mathrm{~ms} / \mathrm{item})$. Using a nulling task, we estimated the speed of illusory rotation in our displays to be approximately $2 \%$. Direct comparison of illusory and real-motion targets moving with matched velocity showed that illusory motion targets were detected more quickly. Blurred target items that conveyed a weak subjective impression of illusory motion gave rise to serial but faster $(<100 \mathrm{~ms} /$ item $)$ search than control items. Our behavioral findings of parallel detection across the visual field, together with previous imaging and neurophysiological studies, suggests that relatively early cortical areas play a causal role in the perception of illusory motion. Furthermore, we hope to reemphasize the way in which visual search can be used as a flexible, objective measure of illusion strength.
\end{abstract}

Keywords Visual search $\cdot$ Visual illusions $\cdot$ Illusory Motion $\cdot$ Attention $\cdot$ Pop-out

\section{Introduction}

Illusions have a long history as useful probes into the functioning of the visual system (Eagleman, 2001; Gregory, 1968; Shapiro \& Todorović, 2016). In recent years there has been much interest in one class of illusion where static images are able to convey a compelling sense of motion. While many different types of "illusory motion" displays have been described and investigated (Bülthoff \& Götz, 1979; Fraser \&

Electronic supplementary material The online version of this article (https://doi.org/10.3758/s13414-019-01750-3) contains supplementary material, which is available to authorized users.

Ian M. Thornton

ian.thornton@um.edu.mt

Sunčica Zdravković

szdravko@f.bg.ac.rs

1 Department of Cognitive Science, Faculty of Media and Knowledge Sciences, University of Malta, Msida, Malta

2 Department of Psychology, Faculty of Philosophy, University of Novi Sad, Novi Sad, Serbia

3 Laboratory for Experimental Psychology, University of Belgrade, Belgrade, Serbia
Wilcox, 1979; Kawahara, Yokosawa, Nishida, \& Sato, 1996; Kitaoka \& Ashida, 2003; Léviant, 1982; Mackay, 1957; Pinna \& Brelstaff, 2000; Gori \& Hamburger, 2006), those popularized by Akiyoshi Kitaoka have proven to be of particular interest to the scientific community (Backus \& Oruç, 2005; Beer, Heckel, \& Greenlee, 2008; Billino, Hamburger, \& Gegenfurtner, 2009; Gori, Agrillo, Dadda, \& Bisazza, 2015; Hisakata \& Murakami, 2008; Otero-Millan, Macknik, \& Martinez-Conde, 2012). Two of Kitaoka's best-known works, "Rotating Snakes" and "Rollers" - works that inspired the original stimuli used in the current experiments - are reproduced in Fig. 1.

Many of Kitaoka's displays - including the examples in Fig. 1 - are directly descended from classic "peripheral drift" illusions (Faubert \& Herbert, 1999; Fraser \& Wilcox, 1979; Naor-Raz \& Sekuler, 2000). Kitaoka has termed these variants "optimized-Fraser-Wilcox illusions," with reference to one early, very influential paper (Fraser \& Wilcox, 1979), as they tend to give rise to a very strong subjective sense of motion. Although the precise mechanisms behind such illusions is still a matter of debate (e.g., Backus \& Oruç, 2005; Conway, Kitaoka, Yazdanbakhsh, Pack, \& Livingstone, 2005; Faubert \& Herbert, 1999; Murakami, Kitaoka, \& Ashida, 2006; Otero-Millan et al., 2012), there is general agreement that the repetitive luminance/contrast gradients 
a) Rotating Snakes

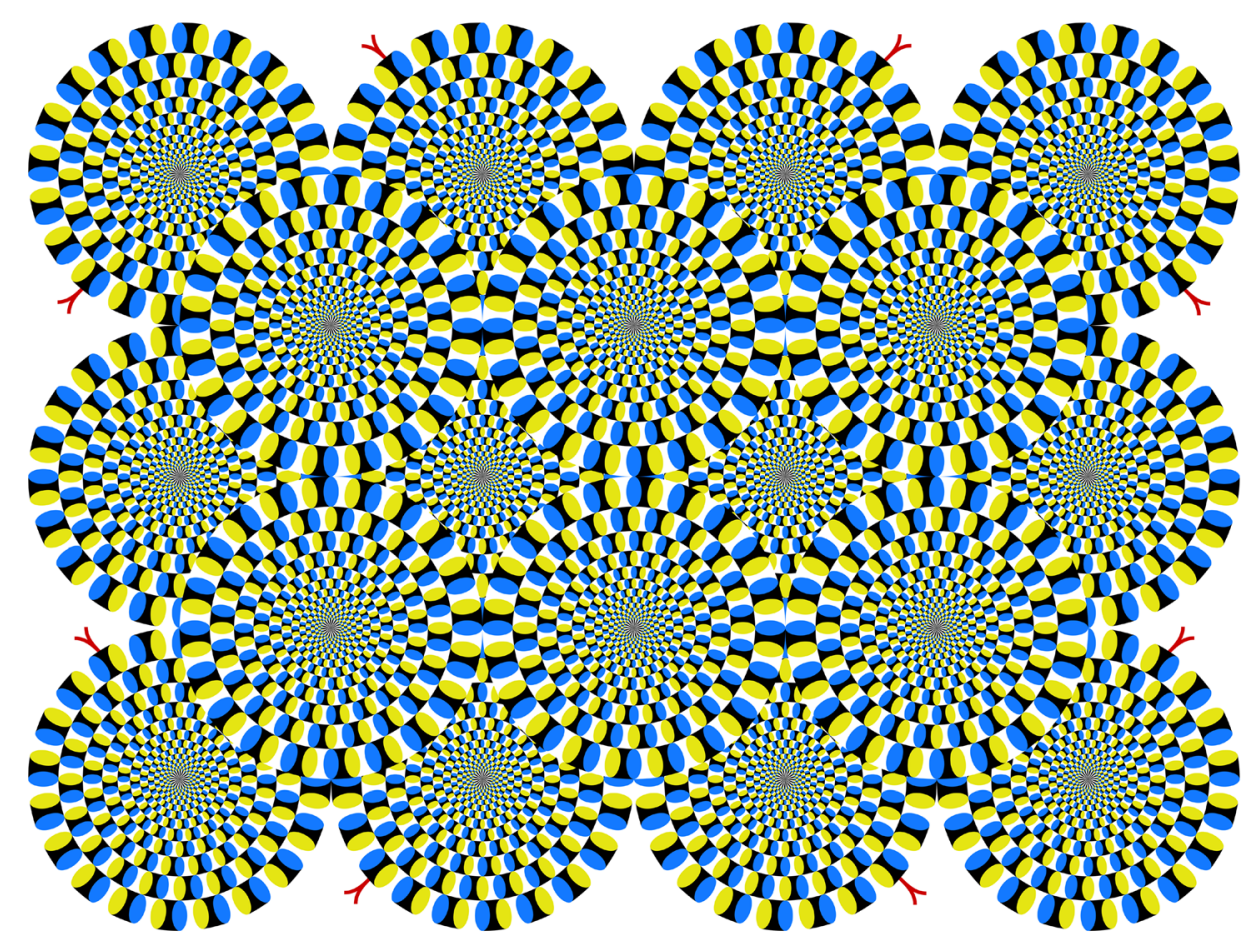

b) Rollers

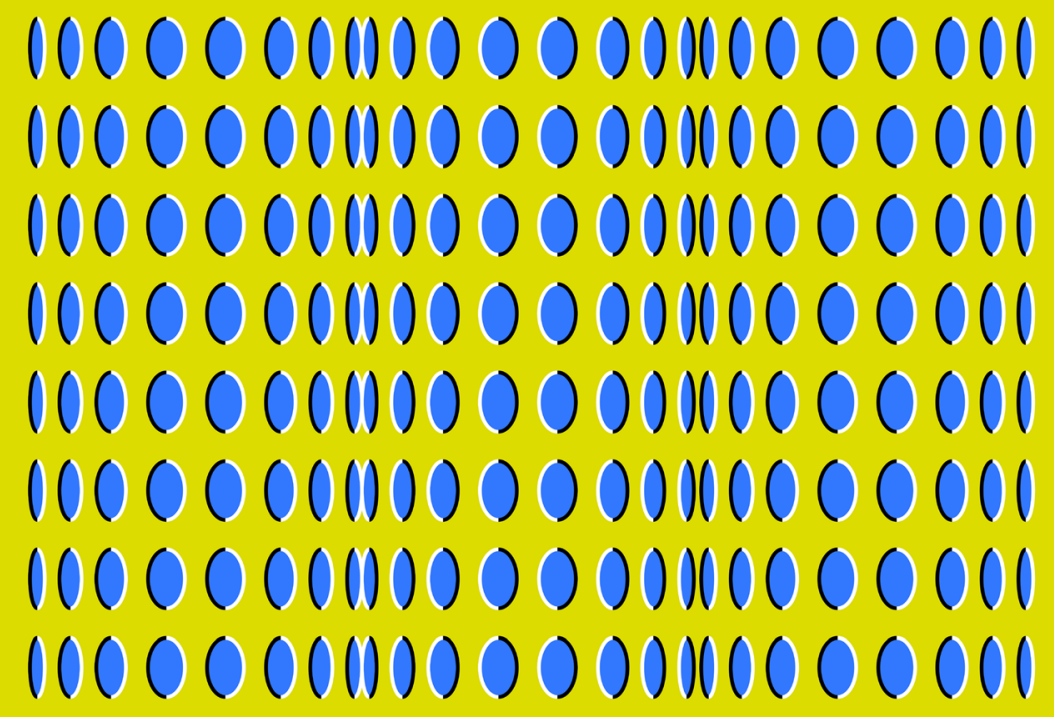

Fig. 1 Two of Akiyoshi Kitaoka's most famous motion illusions, reproduced with permission from the author: (a) Rotating Snakes, (b) Rollers. These variants of the peripheral drift illusion were the inspiration for the stimuli we created for the current study. As the

resonate at some level in the visual system, giving rise to the impression of motion. Indeed, it seems almost certain that the experience of illusory motion activates the same neural illusions work best when images are viewed in high-resolution color, the reader is directed to the online version of this paper or to Kitaoka's excellent webpages, where these and many other examples can be found: http://www.ritsumei.ac.jp/ akitaoka/index-e.html

pathways that are involved in the processing of real motion (Ashida, Kuriki, Murakami, Hisakata, \& Kitaoka, 2012; Conway et al., 2005; Kuriki, Ashida, Murakami, \& 
Kitaoka, 2008; Ruzzoli et al., 2011; Zeki, Watson, \& Frackowiak, 1993).

In the current paper, we use standard visual search methodology (Treisman \& Gelade, 1980; Wolfe, 2010; Wolfe \& Horowitz, 2004, 2017) to further explore the perception of illusory motion. Our main goal was to determine whether the onset of illusory motion pre-attentively guides visual processing in the same way that the onset of luminance-based, real motion is known to do (Abrams \& Christ, 2003; Ashida, Seiffert, \& Osaka, 2001; Dick, Ullman, \& Sagi, 1987; Horowitz, Wolfe, DiMase, \& Klieger, 2007; Nakayama \& Silverman, 1986). Both the compelling subjective phenomenology associated with these illusions - observers typically report concurrent movement across wide areas of the display - and the activation of motion-sensitive cortical regions, suggest that illusory motion could act as such a guide. To our knowledge, however, this idea has never been objectively tested. There are at least two reasons why such an objective test might be useful.

First, as Treisman and Gelade (1980) note in the introduction to their classic paper "A Feature-Integration Theory of Attention," "the immediacy and directness of an impression are no guarantee that it reflects an early stage of information processing in the nervous system. It is logically possible that we become aware only of the final outcome of a complicated sequence of prior operations." (Treisman \& Gelade, 1980 p.98). Treisman and Gelade's use of visual search as an objective measure provided inspiration for countless studies, including Rensink and colleague's search-based approach to "change blindness," perhaps the best recent example of when a subjective impression that we have access to a rich and detailed representation of all before us - can be highly misleading (Rensink, 2000; Rensink, O'Regan, \& Clark, 1997).

Second, activation of a cortical pathway does not, in and of itself, indicate that such activation is causally sufficient to give rise to a specific behavioral pattern. For example, both firstorder (i.e., luminance defined) and second-order (e.g., contrast defined) motion strongly activate human motion pathways, including the human V5/MT+ complex (Ashida, Lingnau, Wall, \& Smith, 2007; Nishida, Sasaki, Murakami, Watanabe, \& Tootell, 2003; Seiffert, 2003; Smith, Greenlee, Singh, Kraemer, \& Hennig, 1998). However, while first-order motion can quickly and efficiently guide attention in a bottom-up fashion (Wolfe \& Horowitz, 2017; Wolfe \& Utochkin, 2019), search for second order motion is "slow and difficult" in nature (Ashida et al., 2001).

To objectively assess the relationship between illusory motion and attention, we conducted a series of four visual search experiments. Experiment 1 examined whether a complex illusory motion target "pops-out" amongst subjectively static distractors. Experiment 2 examined search when targets and distractors had the same structural differences as Experiment 1, but illusory motion was absent. In Experiment 3, we used a nulling technique to estimate the strength of illusory motion on a participant by participant basis, and then compared search for illusory and matched real motion targets. Experiment 4 compared search for illusory motion targets that were either intact, or degraded by blurring.

\section{Experiment 1}

In Experiment 1, participants searched for an illusory motion target amongst a variable set size of perceptually static distractors. The target stimulus, a complex ring composed of 48 atomic elements, is shown in Fig. 2 (upper left). The atomic elements were simple black and white annuli based on the "Rollers" illusion, and they were organized into concentric rings, as in "Rotating Snakes." The overall color scheme, with the yellow background and blue centers, helps provide the crucial contrast gradient for generating the illusion. We should note that in order to clearly experience the illusory rotation, stimuli should be viewed as high-resolution color images. These are available in the online version of this paper, but can also be freely obtained - together with all other experimental material - by contacting the authors.

Subjectively, the target stimulus conveyed a clear sense of slow, clockwise or counterclockwise illusory rotation. The direction of perceived rotation depended on the left/right orientation of the atomic elements, which was counterbalanced across participants. Distractor stimuli contained the identical element and color schemes (Fig. 2, upper right), but with two modifications: First, the left-right organization of consecutive atomic elements was reversed, pitting their local contrast signals against each other; second, the orientation of these atomic elements within a given ring was fixed, thus breaking the global progression that helps imply rotation in the target stimulus. Together, these modifications canceled any subjective sense of local or global illusory motion.

On each trial, elements appeared equally spaced around a virtual circle centered on a permanently visible fixation cross (Fig. 3). Across trials there could be four, five, or six items in a display, with target stimuli appearing on $50 \%$ of the trials. The task was to report the presence/absence of the target using two designated keys (counterbalanced) on a standard USB keyboard. Stimuli remained visible until response. The main dependent variable of interest was the slope of the response time $(\mathrm{RT}) \times$ set size function.

\section{Methods}

\section{Participants}

A total of 12 participants (mean age $=24.4, \mathrm{SE}=1.2$; five female; 11 right-handed) were recruited from the University of Malta academic community. All participants had normal or 


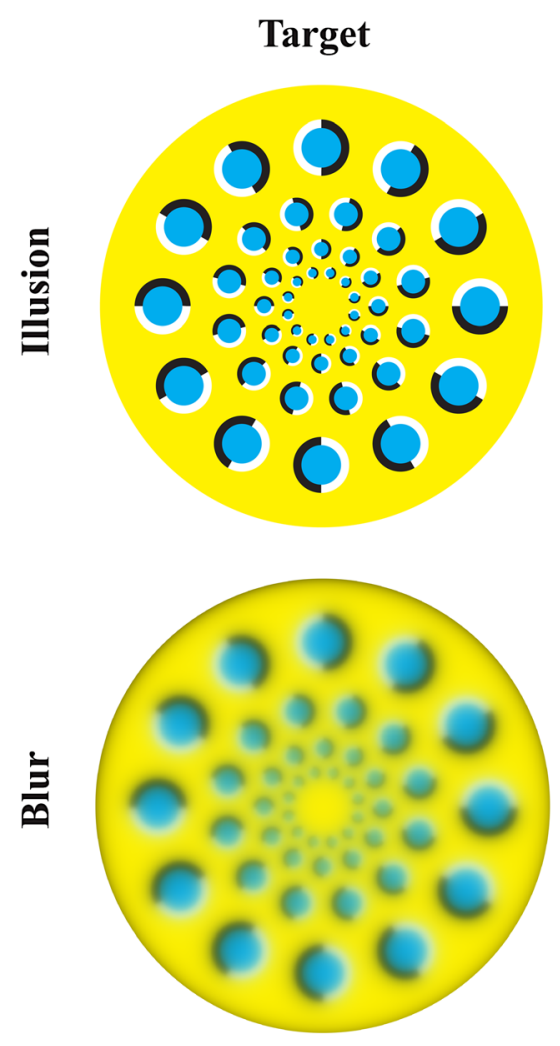

Fig. 2 Target and distractor stimuli created for the current studies. When viewed as color images, the illusion targets (Experiments 1, 3, and 4) convey a clear sense or rotation, either in the clockwise (CW) direction (shown), or, when mirror reversed, in the counterclockwise (CCW) direction. Distractor stimuli have the same local elements, but the

corrected-to-normal vision and gave written informed consent before taking part in the experiment. They were naïve as to the purpose of the research and were not experienced psychophysical observers.

The study was conducted in accordance with the Ethics and Data Protection guidelines of the University of Malta and conformed to the Code of Ethics of the World Medical Association (Declaration of Helsinki).

Group sample size was determined prior to data collection based on previous studies from our group that used visual search with complex, dynamic stimuli (Cavanagh, Labianca, \& Thornton, 2001; Mayer, Vuong, \& Thornton, $2015,2017)$. To verify that this sample size would provide sufficient power to detect search slopes that deviated from zero - our main empirical question - we used the closest matching conditions from this previous work to conduct a priori power analyses. Observed effect sizes (Cohen's d) for the appropriate dynamic conditions were 2.67 (Mayer et al., 2015) and 1.11 (Mayer et al., 2017). Assuming required power of 0.8 and an alpha level of 0.05 , these suggest minimum sample sizes of between four and 11 participants would be sufficient to detect RT $\times$ Set Size functions with non-zero slopes. Thus, our decision to use 12 participants was relatively conservative.
Distractor

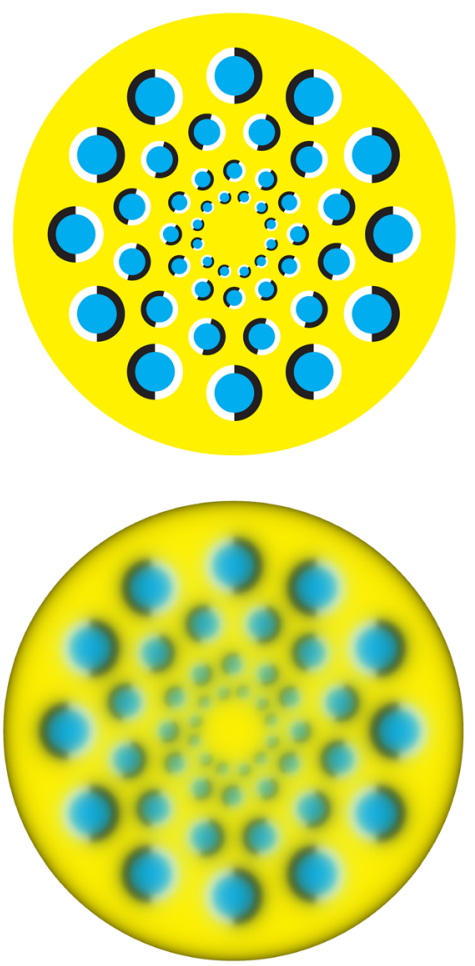

black-white orientation of the atoms reverses at each step around the ring. This eliminates any subjective impression of motion. Blurred targets (Experiment 4) convey a weaker sense of illusory motion due to a reduction in element contrast. See text for more details on stimuli creation and manipulation

\section{Stimuli}

The stimuli used in all experiments were custom designed by the current authors using Adobe Illustrator (Fig. 2). They were closely modelled on two classic illusions by Akiyoshi Kitaoka: Rotating Snakes and Rollers (Fig. 1).

As can be seen in Fig. 2 (upper left), the target stimuli comprised of 48 circular elements organized into four concentric rings. The atomic unit in each ring is a simple annulus whose border has equal black and white parts. The central fill area was always drawn in blue (RGB values: 0/173/239) and the background in yellow (RGB values: 254/242/0). The screen background was set to uniform grey (RGB values: 100). Note that we intentionally report RGB values here, as the presence of illusory motion does not rely on specific display parameters. Indeed, many of these illusions work equally well in printed form as well as on LCD or CRT displays. For the purpose of replication, contrast and brightness simply need to be adjusted so that a compelling subjective sense of illusory motion is present. Nevertheless, for the sake of completeness, in Table S1 of the Supplementary Data, we provide full luminance and colorimetry readings taken during a typical experimental session. 


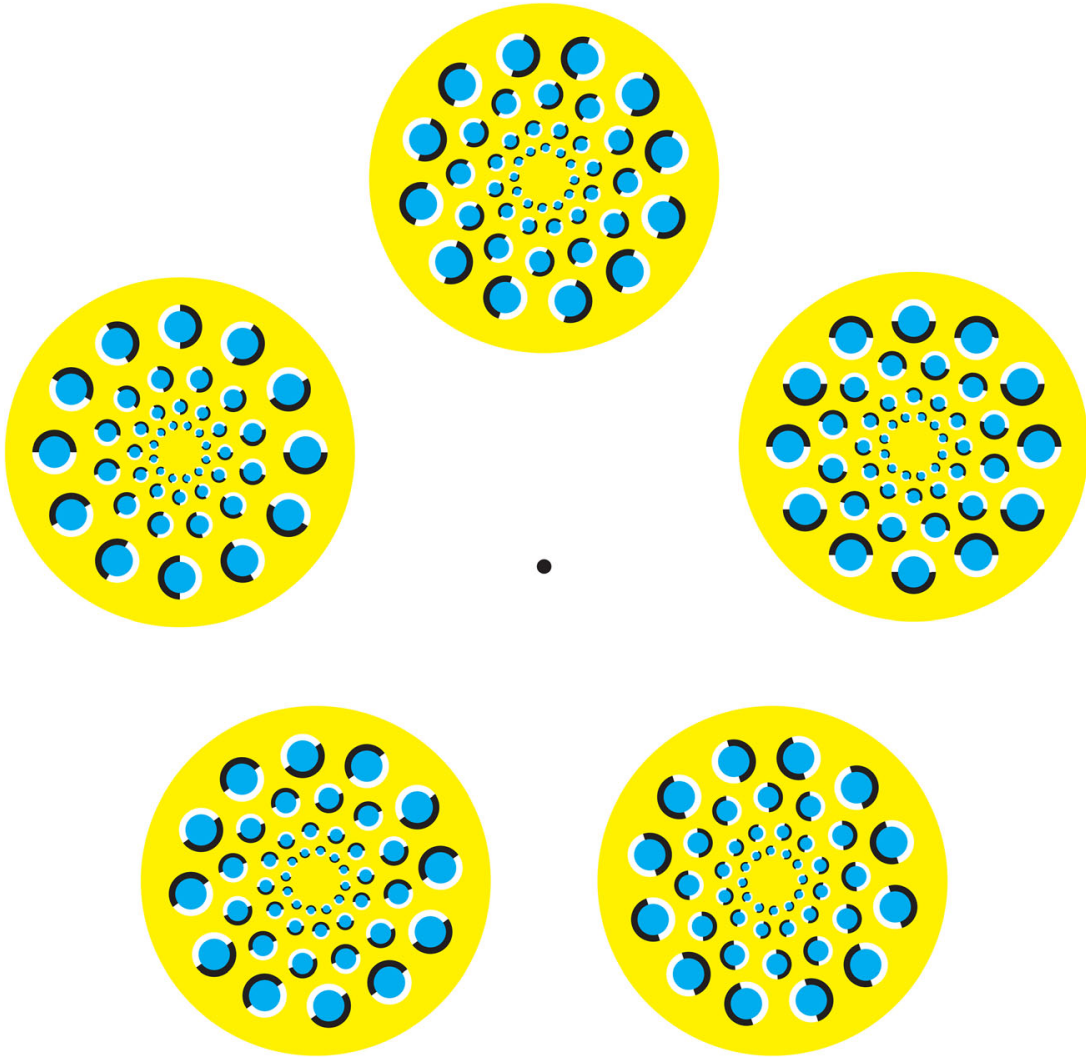

Fig. 3 The circular search array used in Experiments 1, 2, and 3. Participants were asked to fixate on the central dot, and stimuli (four, five, or six items) appeared in equally spaced locations around a virtual

circle. Targets appeared on $50 \%$ of trials and the angular position of the elements was randomly determined on each trial. In this example, the target is present at approximately $270^{\circ}$ or 9 o'clock

To make an individual ring, successive elements were rotated by $30^{\circ}$ around the main circumference and were also locally rotated around their own center by $30^{\circ}$. To make the next smaller ring, the previous version was copied, reduced by $60 \%$, and rotated by $15^{\circ}$. The concentric rings, each with four levels, quite clearly appeared to rotate either clockwise or counterclockwise in the experimental displays. The direction was determined by the order of the black and white border areas. Thus, to make the concentric rings appear to rotate in the opposite direction, the whole image was simply mirror reflected.

For the distractor stimuli (Fig. 2, upper right), the layout and configuration of each ring was exactly the same as for the target stimuli. However, the regular progression of the atomic units in each ring was replaced by a fixed orientation, in which the black and white borders reversed in neighboring atoms. As with the target stimuli, subsequent rings were reduced by $60 \%$ and rotated by $15^{\circ}$ varying the fixed orientation within a ring. This manipulation completely eliminated any sense of illusory motion and mirror reflecting only served to swap the order of black and white elements.

\section{Equipment and laboratory set up}

The experiment was conducted using a 15-in. MacBook Pro, connected to an external BENQ LCD Display. The display had

a visible viewing area of $53 \times 29 \mathrm{~cm}$, and was configured with a resolution of 1,600 $\times 900$ pixels and a simulated refresh rate of $60 \mathrm{~Hz}$. Custom written code was developed in Matlab, using the Psychophysics Toolbox extensions (Brainard, 1997; Kleiner et al., 2007; Pelli, 1997). Copies of the experimental scripts are available on request. Participants sat in a quiet room with no overhead lighting. Viewing distance was approximately $60 \mathrm{~cm}$.

\section{Display layout}

The targets and distractors in both conditions were presented in a circular search configuration (Fig. 3). This ensured that all items were initially presented at the same peripheral eccentricity of approximately $8^{\circ}$ visual angle. On each trial, a small, white fixation point was continuously visible at the center of the screen. Target and distractor images, subtending approximately $7.6^{\circ}$ visual angle, appeared around the circumference of an invisible circle centered at fixation. The individual elements in the outer ring of each stimulus subtended approximately $0.48^{\circ}$ visual angle. The items in each subsequent ring were scaled down by $60 \%$, as described above. The angular position of the target and distractor items around the display circle was randomly determined on each trial, with the constraint that each was separated by $360 / \mathrm{n}$ degrees, where $\mathrm{n}$ is the set size on that given trial. 
On a given trial, there could be four, five, or six items in the display. For each participant, the black-white orientation of the atomic rings - and hence the direction of illusory motion - was fixed, with the orientation being counterbalanced across participants.

\section{Procedure}

After reading written instructions, participants were familiarized with the concept of illusory motion by viewing a series of ten examples, mostly taken from Kitaoka's website. They were then given practice with the main visual search task before proceeding with the experimental blocks of trials. These consisted of 240 trials separated into two blocks, using a 2 (Trial Type: Present or Absent) $\times 3$ (Set Size: 4, 5 or 6$) \times$ 20 (repetition) design. Trial order was determined randomly for each participant, and the whole session lasted approximately 25 mins.

\section{Task}

The task on each trial was simply to determine whether the target stimulus was present or absent. Participants were explicitly asked to base their response on the detection of illusory motion. They were told that maintaining fixation would help to make the illusory motion visible, but we did not monitor eye movements. Responses were made with designated keys on a standard USB keyboard. Target items were present on 50\% of trials. Audible feedback was provided when an error was made and participants were instructed to respond as quickly as possible while keeping the number of errors to a minimum. Stimuli remained visible until response, and no feedback/deadline was provided in terms of response speed.

\section{Data analysis}

Data from one participant could not be analyzed due to a technical error. Our main measure of interest was the slope of the RT $\times$ set size function, which provides a standard way to assess search efficiency. The RT analysis was based only on correct responses (Table 1). We use the median as our point estimate, rather than a normalized/ correct mean, as this is standard practice in our labs and provides a less biased estimate of the underlying RT distributions (see Rousselet \& Wilcox, 2018, for a recent discussion). However, we note that analyses using the mean were qualitatively identical.

Search slopes were calculated for each participant and condition, and average slopes were compared against zero using a one-sample t-test. To more fully characterize the overall pattern of responses, we also conducted a 2 (Target) $\times 3$ (Set Size) repeated-measures ANOVA on the (correct) RT data. Sphericity checks were performed for the appropriate repeated measures factors (i.e., Set Size, Target $\times$ Set Size), but no violations were detected. As accuracy levels remained close to ceiling in all conditions, we did not conduct further analyses, but provide a summary of performance in Table 2 .

\section{Results}

Figure 4 shows average reaction time as a function of set size for both Target present and Target absent trials (see also Table 1). Responses were generally quite slow $\left(\mathrm{M}_{\text {Pres }}=826\right.$ $\mathrm{ms}, \mathrm{SE}=71 ; \mathrm{M}_{\mathrm{Absent}}=1651 \mathrm{~ms}, \mathrm{SE}=275$ ), suggesting that in our complex displays, decision-making required some time to evolve. Importantly, however, when the illusory motion target was present, search was unaffected by the number of distractor elements, giving rise to a flat search slope $(\mathrm{M}=-0.91 \mathrm{~ms} / \mathrm{item}$, $\mathrm{SE}=20$ ) that did not differ from zero, $\mathrm{t}(10)=-0.05$, n.s., $\mathrm{d}=0$. On Target absent trials, search was slower and serial in nature, with slopes $(\mathrm{M}=91 \mathrm{~ms} / \mathrm{item}, \mathrm{SE}=26)$ that were significantly different from zero, $\mathrm{t}(10)=3.3, \mathrm{p}<0.01, \mathrm{~d}=0.99$ (see Table 1).

The two clear patterns that can be seen in Fig. 4 were confirmed using the 2 (Target) $\times 3$ (Set Size) repeatedmeasures ANOVA. First, there was a main effect of Target, with responses on the Target present trials being consistently faster than the Target absent trials, $\mathrm{F}(1,10)=12.0$, MSE $=$ 930314, $\mathrm{p}<.01, \eta_{p}{ }^{2}=0.55$. Second, the Target $\times$ Set Size interaction was significant, $\mathrm{F}(2,20)=5.4, \mathrm{MSE}=9342, \mathrm{p}<$ $.05, \eta_{p}{ }^{2}=0.35$. The main effect of Set Size was not significant, $\mathrm{F}(2,20)=2.2, \mathrm{MSE}=20129$, n.s.

\section{Discussion}

The results of this experiment demonstrate that search for illusory motion is extremely efficient. It would thus seem that, as with luminance-based, real motion, the onset of illusory motion is able to pre-attentively guide vision in parallel across the visual field. To our knowledge, this is the first objective behavioral evidence to back up the compelling subjective impressions and previous neurophysiological and imaging studies that suggested this would be the case with this class of motion illusion.

We note, however, that the target-absent search slopes in Experiment 1 were not flat, as might be expected in simple displays involving static features, such as color, size, and shape. It seems likely that the dynamic nature of the target stimuli - the fact that motion evolves over time - and the overall complexity of the displays could have contributed to this pattern, both affecting participants' criteria for responding on absent trials (Chun \& Wolfe, 1996). For example, participants may have adopted a very conservative (i.e., slow) targetabsent response strategy because the target-present decisions themselves were relatively slow. Also, as only accuracy 
Table 1 Summary of median correct reaction time (RT; ms) as a function of trial type (present/absent), condition (experimental/control) and set size for Experiments 1, 2, and 4

\begin{tabular}{|c|c|c|c|c|c|c|c|c|c|c|}
\hline \multirow[t]{2}{*}{ Exp. 1} & \multicolumn{5}{|l|}{ Present } & \multicolumn{5}{|l|}{ Absent } \\
\hline & 4 & 5 & 6 & Slope & Intercept & 4 & 5 & 6 & Slope & Intercept \\
\hline Illusion & 820 & 840 & 818 & -1 & 831 & 1570 & 1630 & 1751 & 90 & 1197 \\
\hline SEM & 75 & 71 & 76 & 20 & 111 & 265 & 280 & 288 & 27 & 240 \\
\hline \multirow[t]{2}{*}{ Exp. 2} & \multicolumn{5}{|l|}{ Present } & \multicolumn{5}{|l|}{ Absent } \\
\hline & 4 & 5 & 6 & Slope & Intercept & 4 & 5 & 6 & Slope & Intercept \\
\hline Chromatic & 2499 & 2678 & 3042 & 272 & 1382 & 3320 & 3873 & 4470 & 575 & 1014 \\
\hline SEM & 195 & 221 & 233 & 42 & 236 & 215 & 272 & 306 & 80 & 325 \\
\hline Achromatic & 1938 & 2139 & 2489 & 276 & 810 & 2627 & 2896 & 3356 & 364 & 1137 \\
\hline SEM & 118 & 121 & 139 & 46 & 243 & 224 & 264 & 225 & 29 & 273 \\
\hline \multirow[t]{2}{*}{ Exp. 4} & \multicolumn{5}{|l|}{ Present } & \multicolumn{5}{|l|}{ Absent } \\
\hline & 2 & 3 & 4 & Slope & Intercept & 2 & 3 & 4 & Slope & Intercept \\
\hline Illusion & 857 & 794 & 804 & -27 & 953 & 907 & 990 & 1017 & 54 & 670 \\
\hline SEM & 60 & 48 & 52 & 15 & 98 & 53 & 58 & 86 & 20 & 64 \\
\hline Blur & 1121 & 1226 & 1300 & 89 & 768 & 1430 & 1608 & 1951 & 261 & 358 \\
\hline SEM & 96 & 107 & 98 & 23 & 148 & 161 & 157 & 222 & 64 & 287 \\
\hline
\end{tabular}

feedback was provided, this may also have led to target-absent slowing, if participants were actively trying to avoid errors, giving themselves additional time to confirm target absence. Finally, it is important to realize that although luminancebased, real motion is general accepted as a basic visual feature - and as already noted, has been shown to pre-attentively guide attention - the slopes on target absent trials for this type of stimuli are not necessarily flat (Ashida et al., 2001) (see also the results of Experiment 3, below). If target-absent trials for real-motion do not give rise to flat search slopes, we should

Table 2 Summary of accuracy data (\%) as a function of trial type (present/absent), condition (experimental/control) and set size for Experiments 1, 2, and 4

\begin{tabular}{lllllll}
\hline Exp. 1 & Present & \multicolumn{5}{c}{ Absent } \\
& 4 & 5 & 6 & 4 & 5 & 6 \\
Illusion & 95 & 95 & 95 & 98 & 97 & 96 \\
SEM & 2.1 & 2.2 & 2.2 & 2.0 & 2.0 & 2.0 \\
Exp. 2 & Present & & & Absent & \\
& 4 & 5 & 6 & 4 & 5 & 6 \\
Chromatic & 95 & 95 & 95 & 98 & 97 & 96 \\
SEM & 1.8 & 2.0 & 2.0 & 1.5 & 1.6 & 1.6 \\
Achromatic & 94 & 94 & 92 & 98 & 97 & 97 \\
SEM & 1.7 & 1.9 & 1.9 & 1.5 & 1.6 & 1.6 \\
Exp. 4 & Present & & & Absent & & \\
& 2 & 3 & 4 & 2 & 3 & 4 \\
Illusion & 99 & 98 & 99 & 98 & 99 & 99 \\
SEM & 0.4 & 0.6 & 0.5 & 0.6 & 0.5 & 0.3 \\
Blur & 90 & 89 & 85 & 97 & 96 & 96 \\
SEM & 2.8 & 3.3 & 4.2 & 1.2 & 1.9 & 1.7 \\
\hline
\end{tabular}

clearly not expect this to be the case for the respective illusory motion trials.

In Experiment 2, we examine whether efficient targetpresent search in our displays could have been driven by structural differences between targets and distractors, rather than by illusory motion. In Experiment 3, we explore the overall speed of responses. We compare search for illusory motion to search for speed-matched real-motion, to determine whether slow integration is a distinguishing feature.

\section{Experiment 2}

Although the results of Experiment 1 suggest that illusory motion can automatically guide attention, it is important to

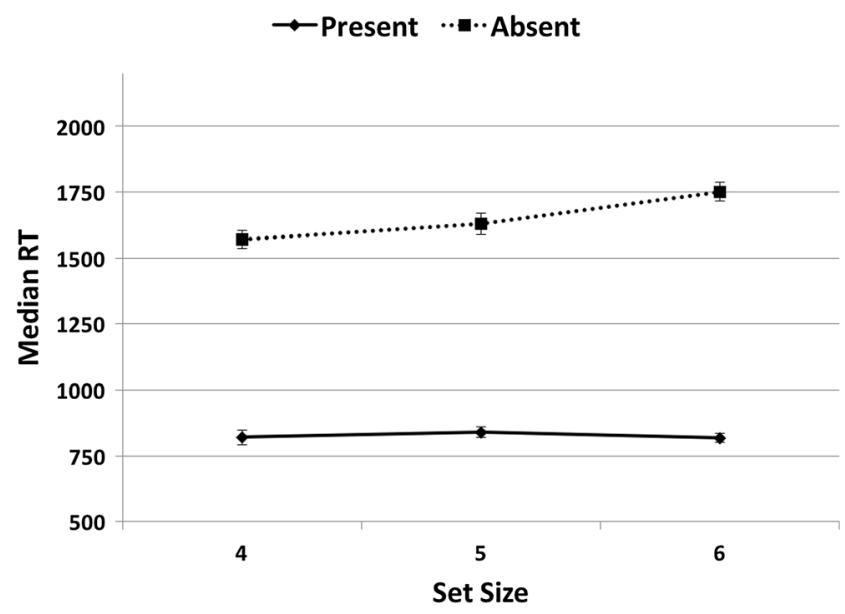

Fig. 4 Search results from Experiment 1. The solid line shows average correct reaction time (RT) for target-present trials, the dashed line for target absent trials. Error bars show standard error of the mean 
explore whether such search behavior could have been driven by structural differences between target and distractor stimuli. To confirm that it was the illusory motion that "popped-out," we ran two control conditions that eliminated the sense of illusory motion, but left the target-distractor structural differences identical to those in Experiment 1.

In the Chromatic Control condition (Fig. 5, top panel), we simply swapped the position of the blue and white segments in both Target and Distractor items. Even though these stimuli share the same structure and color elements of the stimuli used in Experiment 1, in breaking the crucial yellow-black-bluewhite color contrast gradient the subjective sense of illusory motion is eliminated.

As discussed in more detail below, a further side-effect of shuffling the color contrast elements in this way it to reduce the apparent saliency of the border elements. This is clearly visible in Fig. 5, where the white central area of the Chromatic Control seems to dominate, giving the (illusory) impression that the blue-black border elements are smaller, compared to the illusory motion stimuli. As such reduced border saliency could affect search behavior, we also ran a further Achromatic Control condition (Fig. 5 , bottom panel). In this condition, the structure is again identical to the stimuli used in Experiment 1, but we only

Target
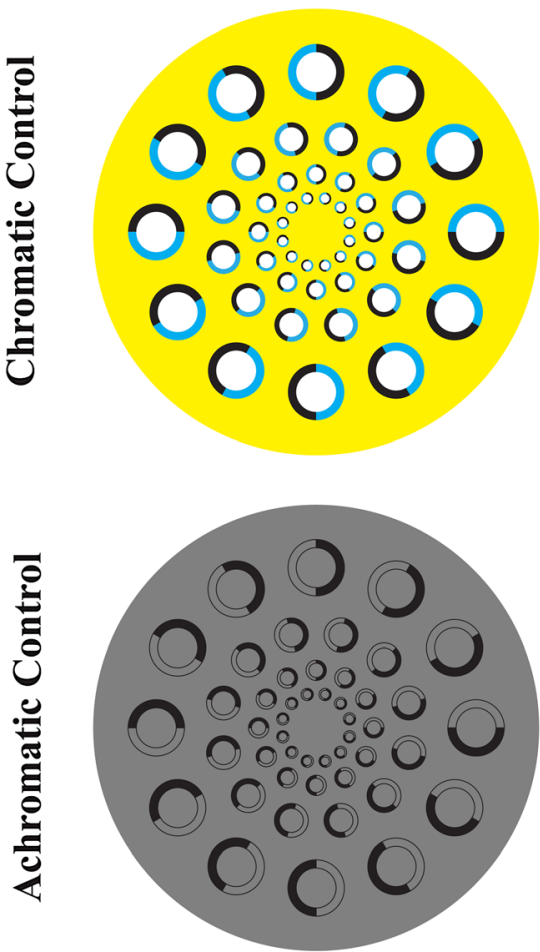

Fig. 5 Control target and distractor stimuli created for Experiment 2. Note that these stimuli are structurally identical to those in Fig. 2. In particular, differences between the targets and distractors have been completely maintained. Chromatic Control-only stimuli differ from the retained the solid black elements of the display and the black outlines. Again, there is no sense of illusory motion in the target stimuli, but in this condition, there is no reduction in the clarity of structural differences between target and distractor items.

Our prediction for both control conditions was that with the illusory motion removed, search would be slow and serial for both target present and target trials.

\section{Methods}

\section{Participants}

A total of 24 new participants took part in this study. Twelve participants completed the Chromatic Control condition, and were recruited from the University of Malta community (mean age $=26.3, \mathrm{SE}=1.0$; four female; all right-handed). A further 12 participants (mean age $=19.8, \mathrm{SE}=1.5$; eight female; 11 right-handed), completed the Achromatic Control Condition and were recruited from the Department of Psychology subject pool at the University of Novi Sad, Serbia. Group sample size was determined prior to data collection as described in Experiment 1. All participants had normal or corrected-tonormal vision and gave written informed consent before

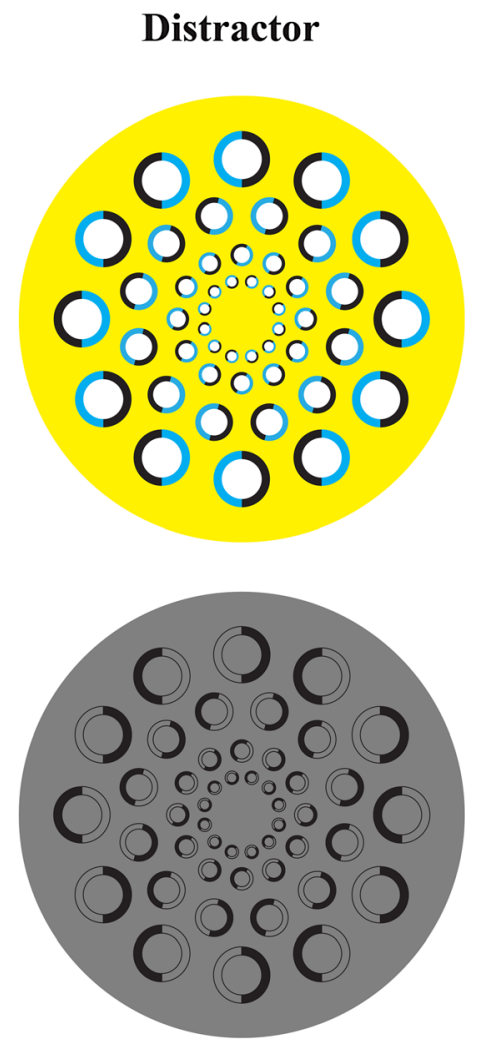

Illusion stimuli in that the blue and white segments have been swapped. The Achromatic stimuli contain only black and gray elements, but also have an identical structure to the Illusion stimuli. Subjectively, neither of the control targets convey any sense of motion 
taking part in the experiment. They were naive as to the purpose of the research and were not experienced psychophysical observers.

The study was conducted in accordance with the Ethics and Data Protection guidelines of the University of Malta and the University of Novi Sad, Serbia, and conformed to the Code of Ethics of the World Medical Association (Declaration of Helsinki).

\section{Stimuli}

The target and distractor stimuli used in Experiment 2 are shown in Fig. 5. Note that for both the Chromatic Control condition and the Achromatic Control condition the stimuli were structurally identical to those used in Experiment 1 (top row, Fig. 2). To create the Chromatic Control stimuli, we simply swapped the position of the blue and white components in each part of the display. This disrupted the crucial yellow-black-blue-white color contrast gradient that is thought to give rise to the sense of illusory motion, but did not alter the basic shape, nor the structural difference between target and distractor stimuli. Subjectively, as intended, there is no sense of illusory motion in either the target or distractor in this condition.

However, as already mentioned, shifting the highest luminance component to the center of the rings does appear to reduce the structural salience of the individual atomic elements of the display, in addition to removing the sense of illusory motion. This is particularly obvious in the distractor items, where the alternation in the border elements is much harder to see than in the original (Experiment 1) version. Indeed, it appears that the border elements are much narrower in the Chromatic Control display, where in fact they are identical.

As this reduction in saliency could also have a clear effect on target detection, we created a second Achromatic Control - condition, in which we removed all color contrast. Again, this eliminated any sense of illusory motion, while leaving the structure of the displays identical to those in Experiment 1. Here, however, the structural differences between targets and distractors appears much less affected.

\section{Task}

Given the absence of any subjective sense of illusory motion in these displays, the task instructions and familiarization process were necessarily different. That is, the structural differences between the target and distractor items were explicitly shown, and these were identified as the basis for response decisions.

\section{Equipment, display, procedure, and data analysis}

All other aspects of Experiment 2 were identical to those described in Experiment 1.

\section{Results}

The full pattern of reaction data for the Chromatic and Achromatic conditions are provided in Table 1. Figure 6 compares the search slopes of RT $\times$ Set Size functions to that found during search for illusory motion in Experiment 1. It is clear that search for both types of control target item were extremely slow and serial in nature. Target present slopes in both Chromatic $(\mathrm{M}=272$ $\mathrm{ms} /$ item, $\mathrm{SE}=42)$ and Achromatic $(\mathrm{M}=276 \mathrm{~ms} /$ item, SE =46) conditions were significantly different from zero: Chromatic Present, $\mathrm{t}(11)=6.5, \mathrm{p}<0.001, \mathrm{~d}=1.9$; Achromatic Present, $\mathrm{t}(11)=6.0, \mathrm{p}<0.001, \mathrm{~d}=1.7$. The respective Target absent slopes were also significantly different from zero: Chromatic Absent $\mathrm{t}(11)=7.2, \mathrm{p}<0.001$, $\mathrm{d}=2.1 ;$ Achromatic Absent, $\mathrm{t}(11)=12.7, \mathrm{p}<0.001, \mathrm{~d}=$ 3.7.

To further explore the pattern of RT data we also conducted a 2 (Condition) $\times 2$ (Target) $\times 3$ (Set Size $)$ mixed ANOVA. There was a main effect of Condition, reflecting the fact that responses in the Chromatic condition $(\mathrm{M}=$ $3314 \mathrm{~ms}, \mathrm{SE}=192)$, were consistently slower than the Achromatic condition $(\mathrm{M}=2574 \mathrm{~ms}, \mathrm{SE}=192), \mathrm{F}(1,22)$ $=7.4, \mathrm{MSE}=2662529, \mathrm{p}<.05, \eta_{p}{ }^{2}=0.25$. Although there were main effects of both Target, $\mathrm{F}(1,22)=64.4$, $\mathrm{MSE}=515242, \mathrm{p}<.001, \eta_{p}{ }^{2}=0.74$ and Set Size, $\mathrm{F}(2,44)=90.6, \mathrm{MSE}=74048, \mathrm{p}<.001, \eta_{p}{ }^{2}=0.8$, these need to be interpreted in the context of a significant Target $\times$ Set Size interaction, $\mathrm{F}(2,44)=8.1$, MSE $=$

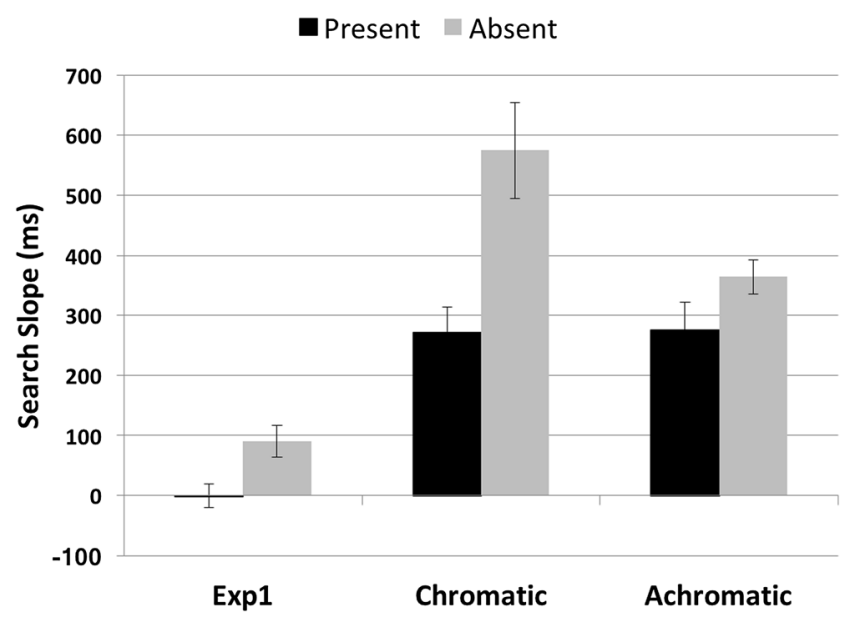

Fig. 6 Search slopes from Experiment 2. Summary of the target-present and target absent search slopes from the two conditions of Experiment 2. The corresponding slopes from Experiment 1 are also shown for comparison. See Table 1 for details of the RT $\times$ Set Size functions. Error bars show standard error of the mean 
56894, $\mathrm{p}<.01, \eta_{p}{ }^{2}=0.27$. RTs increased more steeply as function of Set Size in Target Absent trials. No other interactions were significant.

\section{Discussion}

Search in the absence of illusory motion was slow and serial in nature, with the overall speed of responses suggesting that multiple fixations were probably required to inspect individual items. These findings indicate that the efficient search seen in Experiment 1 did not arise due to structural difference between the target and distractor items. The overall slower responses in the Chromatic condition may have arisen due to the phenomenal reduction in border saliency that was discussed above. However, as this was a between-subjects design, and the participant demographics varied in terms of age and gender, we cannot rule these out as contributing factors.

Examination of Fig. 6 further suggests that the slowing in the Chromatic condition may be mainly driven by different behavior in the target-absent trials. Target present search slopes were quite comparable across the two conditions/groups (see Table 1). Again, this Target absent pattern could reflect greater difficulty in distinguishing targets from distractors in the Chromatic condition or a more conservative approach in sampling the display from that group of participants.

\section{Experiment 3}

The results of Experiment 1 are consistent with the idea that the onset of illusory motion can be detected in parallel across the visual field as is the case for the onset of real-motion. As already noted, however, target present responses were generally quite slow, with an average RT $\times$ Set Size intercept of approximately $800 \mathrm{~ms}$ (see also Table 1). The purpose of Experiment 3 was to directly compare search for illusory motion targets to that of real-motion targets that were physically rotating at a comparable speed. To achieve this, four experienced psychophysical observers first completed a nulling task that allowed us to obtain individual estimates of illusory rotation speed. The methodology was adapted from the work of Hisakata and Murakami (2008), who used the same nulling task to measure changes in illusion strength as a function of eccentricity and retinal illuminance. Following the nulling task, the same four observers then completed a search task. This was identical to that used in Experiment 1 , except that illusory and individual matched real-motion targets were interleaved randomly within blocks of trials. We expected search slopes for both types of targets to be flat. Our question was whether search speeds for the two types of motion stimuli differed in any consistent way.

\section{Methods}

\section{Participants}

Four experienced psychophysical observers (two authors) took part in this study (mean age $=54, \mathrm{SE}=4.8$; one female; four right-handed). All had normal or corrected-to-normal vision and gave informed consent before taking part in the study. We based the sample size on standard psychophysical methods which typically employ large numbers of measurements taken from small groups of experienced observers (see Normand, 2016; Smith \& Little, 2018 for recent discussions).

The study was conducted in accordance with the Ethics and Data Protection guidelines of the University of Malta and the University of Novi Sad, Serbia, and conformed to the Code of Ethics of the World Medical Association (Declaration of Helsinki).

\section{Equipment and laboratory set up}

For three of the observers, the equipment and lab set up were the same as that used in Experiment 1. The fourth observer (QCV), ran in a different laboratory setting, but with the same monitor and comparable viewing conditions. We note that in both experimental tasks, a central fixation point was always visible at the center of the display, and all observers reported consistently maintaining fixation during the trials.

\section{Nulling task}

On each trial of the nulling task, a single target stimulus appeared for $500 \mathrm{~ms}$ at a random location around an $8^{\circ}$ eccentric viewing circle, centered on fixation. Target stimuli were those used in Experiment 1 (Fig. 2). Once the target disappeared, the observer was asked to report its direction of rotation, using their right index finger on the "l" key for a CW response, and their left index finger on the "s" key for a CCW response.

There were two, independent, randomly interleaved target sequences, one containing the $\mathrm{CW}$ illusory target from Experiment 1 and the other containing the CCW illusory target from Experiment 1. Using the method of constant stimuli, we parametrically varied the physical rotation speed of the stimuli within these streams within the range -4 to $4 \%$, where negative values indicate $\mathrm{CCW}$ rotation. The full motion range contained 16 steps corresponding to $0.5 \%$ increments, although individual observers were only presented with 11 steps, depending on initial estimates of their motion sensitivity (see Fig. 7 for details). Each observer completed four blocks of 110 trials, providing 20 responses for each data point. 


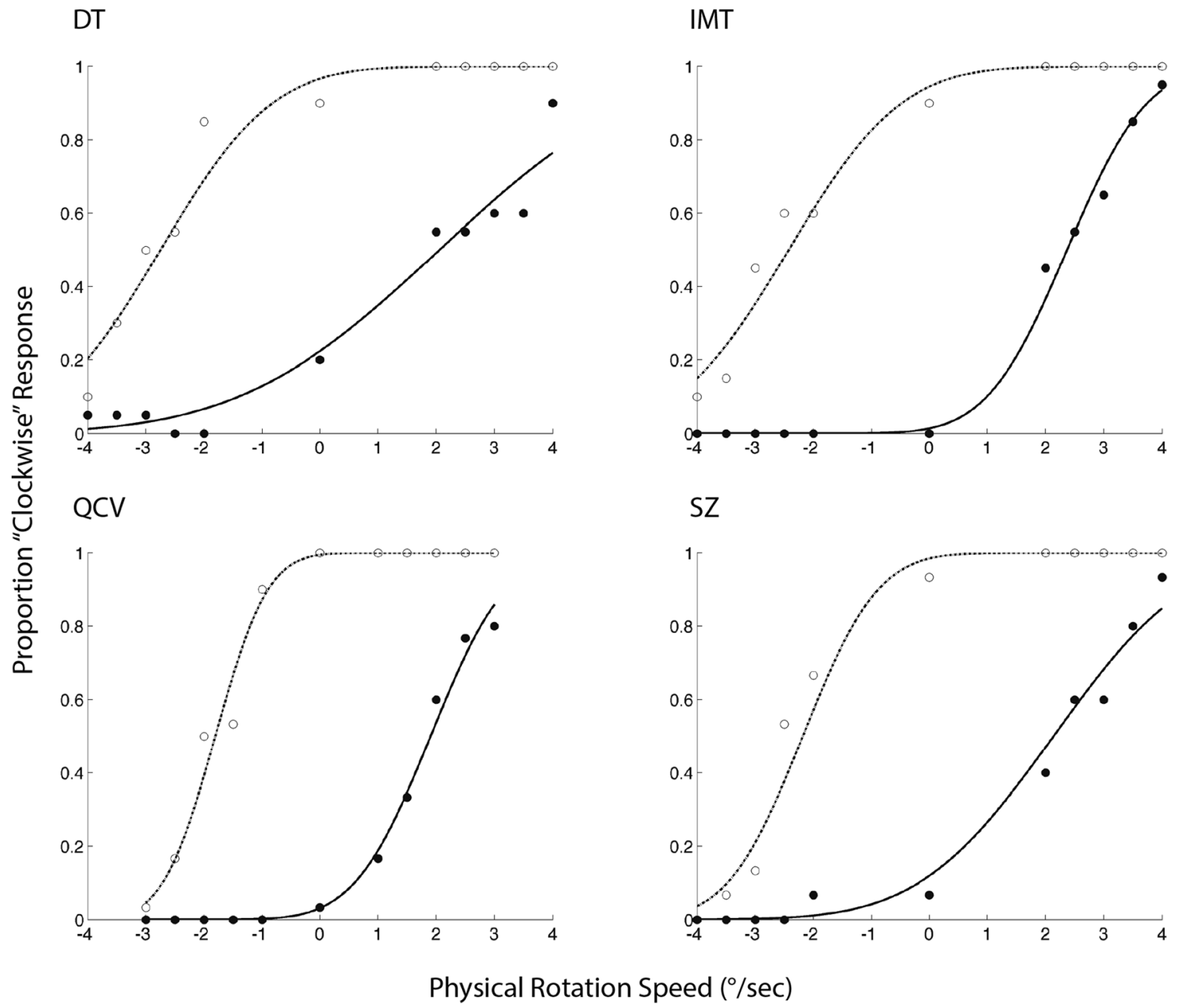

Fig. 7 Raw data and psychometric functions for each of the observers in the nulling task of Experiment 3. Open symbols (dashed line) show responses when a CW illusory was present, closed symbols (solid line) for the CCW illusion. See Table 3 for estimates of PSS and goodness of fit

We estimated the point of subjective stationarity (PSS) separately for the two motion streams of each observer by fitting cumulative normal distributions to the raw data. Curve fitting was performed using the Palamedes Toolbox for MATLAB (Prins \& Kingdom, 2018). Goodness of fit was determined via the bootstrapping method described by Wichmann and Hill (2001). To obtain an overall speed estimate we followed Hisakata and Murakami (2008) and divided the distance between the PSSs for the CW and CCW target streams by two.

\section{Search task}

The design of the search task was identical to that used in Experiment 1, with a single exception. Target present trials could now contain either real or illusory motion stimuli. These target present trials were interleaved randomly with the target absent trials and the overall trial order was randomly assigned within a block of each observer independently. On real-motion target-present trials, a distractor item (Fig. 2) was

Table 3 Summary of the psychometric curve fitting for each of the observers in the nulling task of Experiment 3

\begin{tabular}{llcccccc}
\hline Observer & PSS (CCW) & Pdev & pValue & PSS (CW) & Pdev & pValue & Speed estimate \\
\hline DT & 2.05 & 10.71 & 0.34 & -2.75 & 6.46 & 0.21 & 2.40 \\
IMT & 2.37 & 1.71 & 0.83 & -2.43 & 4.11 & 0.44 & 2.40 \\
QCV & 1.90 & 1.85 & 0.82 & -1.81 & 4.40 & 0.36 & 1.85 \\
SZ & 2.01 & 12.34 & 0.13 & -2.18 & 5.36 & 0.26 & 2.10 \\
\hline
\end{tabular}


Table 4 Mean correct reaction time (RT; ms) for each observer in the search task of Experiment 3 as a function of trial type (present/absent), motion type (illusion/real), and set size

\begin{tabular}{|c|c|c|c|c|c|c|c|c|c|c|}
\hline \multirow[t]{2}{*}{ DT } & \multicolumn{5}{|c|}{ Present } & \multicolumn{5}{|c|}{ Absent } \\
\hline & 4 & 5 & 6 & Slope & Intercept & 4 & 5 & 6 & Slope & Intercept \\
\hline Illusion & 814 & 828 & 798 & -8 & 851 & 971 & 975 & 1032 & 31 & 838 \\
\hline SEM & 22 & 21 & 17 & & & 29 & 23 & 26 & & \\
\hline Real & 956 & 909 & 953 & -1 & 946 & 954 & 997 & 1010 & 28 & 847 \\
\hline SEM & 30 & 34 & 25 & & & 26 & 28 & 20 & & \\
\hline \multirow[t]{2}{*}{ IMT } & \multicolumn{5}{|c|}{ Present } & \multicolumn{5}{|c|}{ Absent } \\
\hline & 4 & 5 & 6 & Slope & Intercept & 4 & 5 & 6 & Slope & Intercept \\
\hline Illusion & 517 & 515 & 503 & -7 & 546 & 615 & 645 & 673 & 29 & 500 \\
\hline SEM & 9 & 13 & 7 & & & 14 & 16 & 15 & & \\
\hline Real & 600 & 581 & 589 & -6 & 619 & 627 & 651 & 674 & 24 & 532 \\
\hline SEM & 14 & 17 & 15 & & & 14 & 17 & 16 & & \\
\hline \multirow[t]{2}{*}{ QCV } & \multicolumn{5}{|c|}{ Present } & \multicolumn{5}{|c|}{ Absent } \\
\hline & 4 & 5 & 6 & Slope & Intercept & 4 & 5 & 6 & Slope & Intercept \\
\hline Illusion & 477 & 484 & 474 & -2 & 487 & 567 & 601 & 599 & 16 & 510 \\
\hline SEM & 11 & 14 & 13 & & & 17 & 25 & 22 & & \\
\hline Real & 614 & 575 & 579 & -17 & 676 & 585 & 631 & 639 & 27 & 483 \\
\hline SEM & 26 & 13 & 12 & & & 16 & 16 & 20 & & \\
\hline \multirow[t]{2}{*}{ SZ } & \multicolumn{5}{|c|}{ Present } & \multicolumn{5}{|c|}{ Absent } \\
\hline & 4 & 5 & 6 & Slope & Intercept & 4 & 5 & 6 & Slope & Intercept \\
\hline Illusion & 644 & 658 & 640 & -2 & 657 & 751 & 785 & 767 & 8 & 729 \\
\hline SEM & 16 & 22 & 20 & & & 24 & 34 & 19 & & \\
\hline Real & 814 & 758 & 819 & 3 & 784 & 715 & 782 & 777 & 31 & 604 \\
\hline SEM & 26 & 20 & 25 & & & 23 & 26 & 24 & & \\
\hline
\end{tabular}
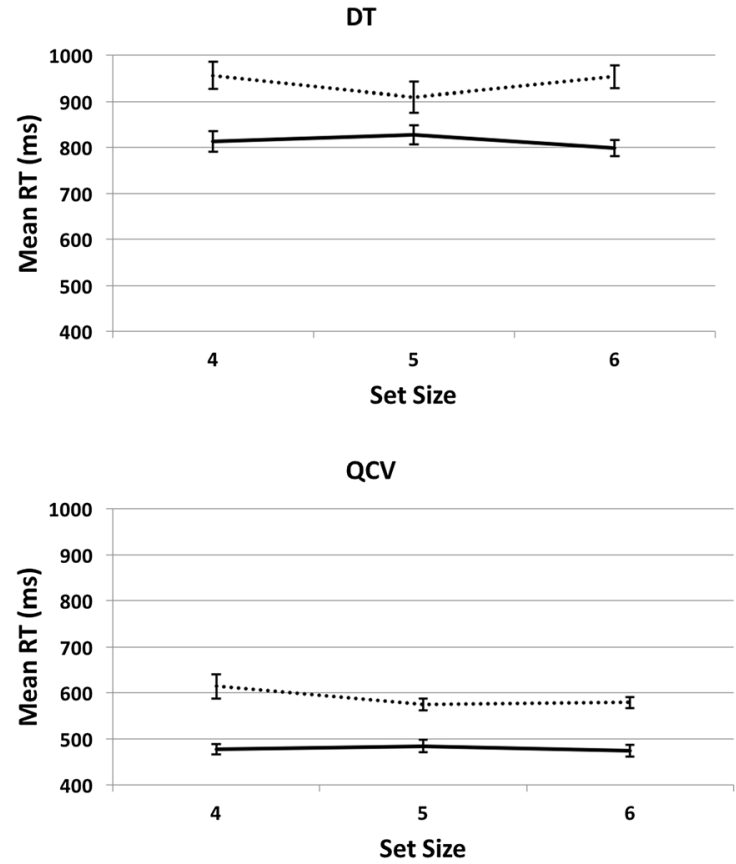

Fig. 8 Search performance for Experiment 3. Data show the targetpresent RT $\times$ Set Size functions for each observer separately as a function of motion type. Solid lines are the Illusion condition, dashed
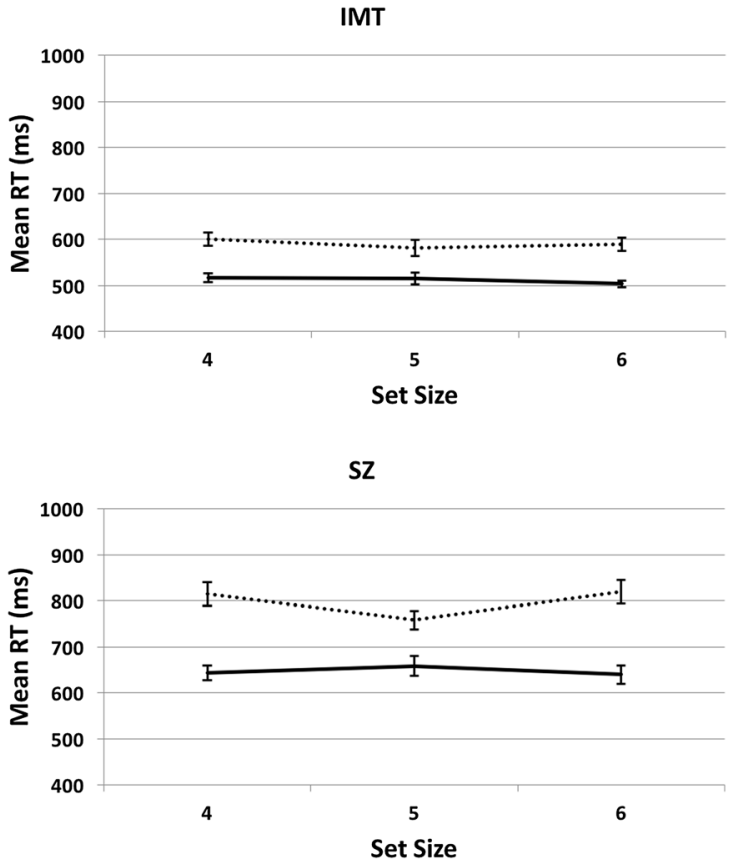

lines the Real Motion condition. Error bars show standard error of the mean. See Table 4 for other search parameters 
made to physically rotate. The speed of physical rotation was fixed and independently determined for each observer based on their corresponding nulling task results. During the search task, and following the design of Experiment 1, target motion - both illusory and real - was only seen in a single direction. Target direction was randomly assigned for each observer. The task was simply to report the presence or absence of motion, and the key assignment for a target-present response was kept consistent with the mapping used during the nulling task.

All observers completed six blocks of 120 trials using a 2 $($ present/absent $) \times 2($ motion type $) \times 3($ set size $) \times 10$ (repetition) design. Data analysis procedures were the same as those in Experiments 1 and 2, although here we report mean values for the $\mathrm{RT} \times$ set size functions in order to better capture individual participant variability. Note that patterns of data were qualitatively identical when using the median as the point estimate of central tendency. As accuracy was essentially at ceiling for all observers, we do not present those data.

\section{Results}

Figure 7 shows the raw data and psychometric functions for each of our four observers. The probability of reporting $\mathrm{CW}$ motion is plotted as a function of the physical rotation speed, with the positive values corresponding to actual clockwise rotation. It is clear that for all four observers, illusory motion had a strong influence on responses. When the physical rotation speed was zero, responses were entirely determined by illusory motion. Furthermore, at almost all physical speed levels, the presence of the $\mathrm{CW}$ illusion led to more $\mathrm{CW}$ responses than when the CCW illusion was present (and vice versa). Table 3 summaries the results of fitting cumulative normal distributions to these data. We provide the PSS for each illusion direction, estimates of goodness of fit, and an overall estimate of illusory speed obtained for each observer.

All curves had acceptable goodness of fit estimates. PSS values were consistently shifted away from zero in the direction opposite to the illusory motion (see also Fig. 7). For example, to appear stationary, observer DT required a CWW illusion to physically rotate in the $\mathrm{CW}$ direction at approximately $2.05 \%$ s. For all observers, the shifts were essentially symmetrical for the two illusion directions. Across observers, the resulting magnitude of the estimated nulling speeds were very consistent, with a mean rotation speed of just over $2 \%$ $(\mathrm{M}=2.2, \mathrm{SE}=0.3)$.

Table 4 summarizes the RT $\times$ set size data for correct trials in the search task for each observer. Separate rows show the

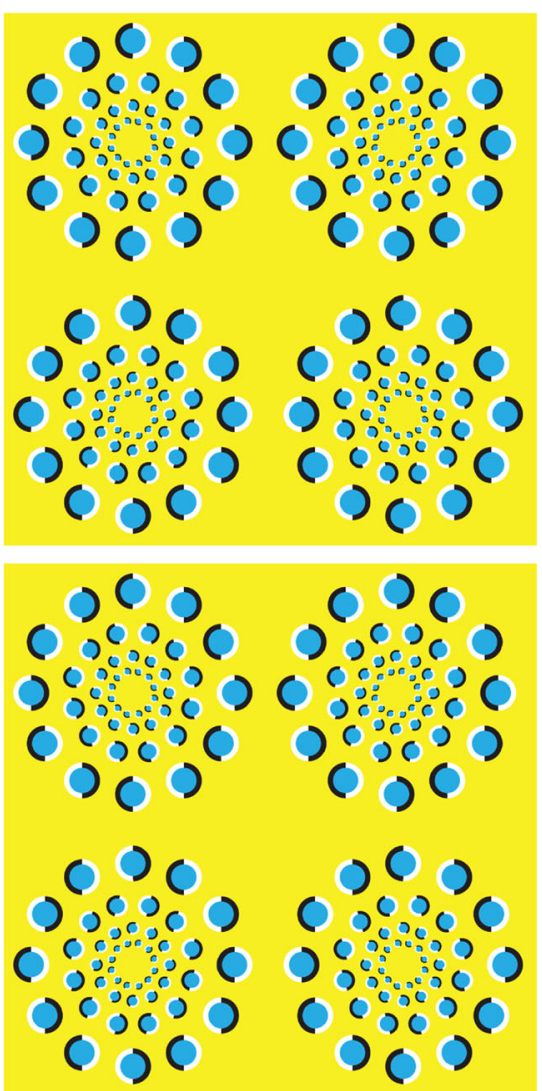

Fig. 9 The grid layout used in Experiment 4. Target and distractor stimuli consisted of quartets made from four of the basic elements used in Experiment 1. Set size could be two, three, or four quartets, and targets
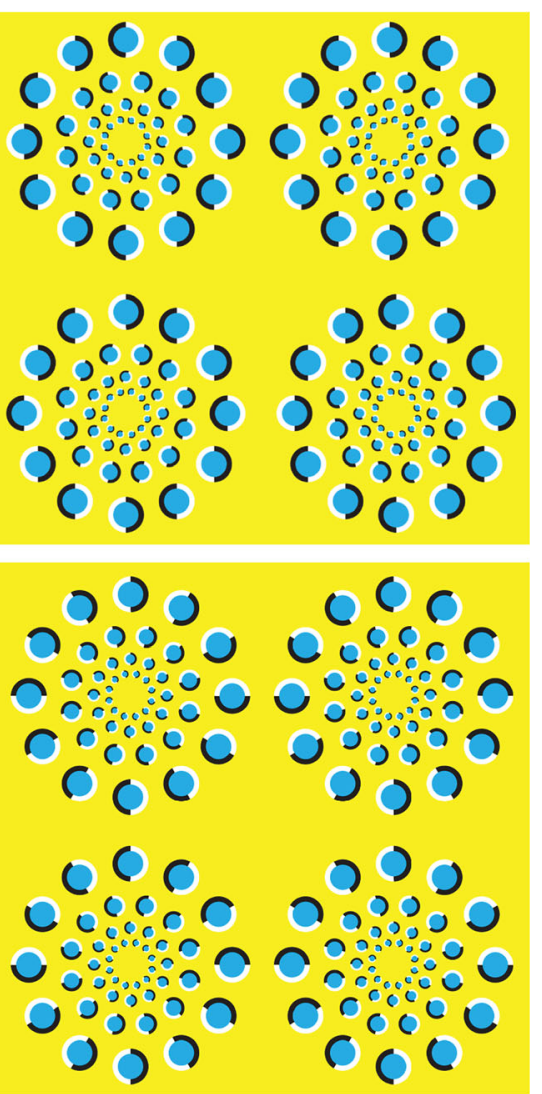

were presented on $50 \%$ of trials. In this example, the target is present in the lower right corner. See text for more details on quartet design 
data for trials that contained illusory motion and real motion. As expected, the slopes for all target-present functions are approximately zero, verifying that illusory motion, as well as real-motion, is able to pre-attentively guide visual processing. Our main interest in the current experiment was the relative speed with which the two types of motion were detected. These patterns are shown in Fig. 8, which plots targetpresent RT as a function of set size for both illusory (solid lines) and real (dashed lines) motion. As can clearly be seen, for all four observers, illusory motion $(\mathrm{M}=613 \mathrm{~ms}, \mathrm{SE}=76)$ was detected more quickly than real motion $(\mathrm{M}=729 \mathrm{~ms}$, SE $=86), \mathrm{t}(3)=7.8, \mathrm{p}<0.01, \mathrm{~d}=3.9$.

\section{Discussion}

There were several interesting findings from Experiment 3. First, we were able to estimate the apparent speed of rotation of the illusory target stimuli used in Experiment 1. The estimate of around $2 \%$ was relatively consistent across the four observers. Although this represents very slow motion, we note that these estimates are an order of magnitude larger than those measured by Hisakata and Murakami (2008) at comparable eccentricities. Their use of simplified, grayscale stimuli, which are known to give rise to weaker subjective impressions of rotation, probably accounts for this discrepancy (see their Figs. 1 and 4).

Second, we found that the relatively slow detection speeds noted in Experiment 1 are not specific to illusory motion targets. Rather, such detection times seem to reflect the sluggish processing of slow motion in the context of a complex display. Indeed, for the current four observers, illusory motion targets were actually found more quickly than real-motion targets. We return to this latter finding, and what it might tell us about the mechanisms that support illusory motion perception, in the General discussion.

Third, we should note that all of the observers reported being unable to subjectively distinguish between the illusory motion and real-motion target-present trials in the context of the speeded search task. That is, they responded based on the impression that "something" was moving, not on the basis of "what" was moving. Almost certainly, in an unspeeded task, once attention had been deployed, the clear structural difference between the illusory motion target and the rotating distractor would become apparent. Nevertheless, the initial lack of a clear qualitative difference between the two types of motion is intriguing and clearly warrants further study.

As a final point, we note that absolute detection speeds in Experiment 3 were considerably faster than those observed in Experiment 1, although as can be seen in Fig. 8, our experienced observers did vary somewhat in terms of absolute speed. We attribute the generally faster responses to our use of experienced observers in contrast to the naïve participants used in Experiment 1. Quite possibly, if we had provided response-time feedback or had encouraged more rapid responding by the use of brief displays or response deadlines, we could have seen faster responses in Experiment 1 . However, even for the fastest observer in Experiment 3, detection times would not be considered rapid, which almost certainly reflects the overall complexity of the displays and slow nature of the motion.

\section{Experiment 4}

The goal of our final experiment was to replicate the illusory motion search patterns seen in Experiments 1 and 3 using a display that more closely resembled Kitaoka's original illusions. Rather than presenting peripheral stimuli, we asked participants to search for illusory motion "quartets" that appeared in the presence of one, two or three distractor quartets organized in a centrally presented grid. Figure 9 illustrates these quartets and also the grid layout of the search arrays, with the maximum set-size of four items. In contrast to the previous experiments, we did not provide a central fixation point, and participants were instructed to free-view the displays.

In Experiment 2, the target stimuli in the Chromatic and Achromatic control conditions were designed to remove any sense of motion. We showed clearly that search for such targets - which had identical structurally differences to their distractors - was highly inefficient. In Experiment 4, rather than trying to eliminate the sense of illusory motion, we decided to use a control condition where we simply weakened it. To do this, we used low-pass filtered versions of the illusory targets, blurring the crucial contrast borders. Subjectively, such blurred stimuli give rise to a much weaker sense of

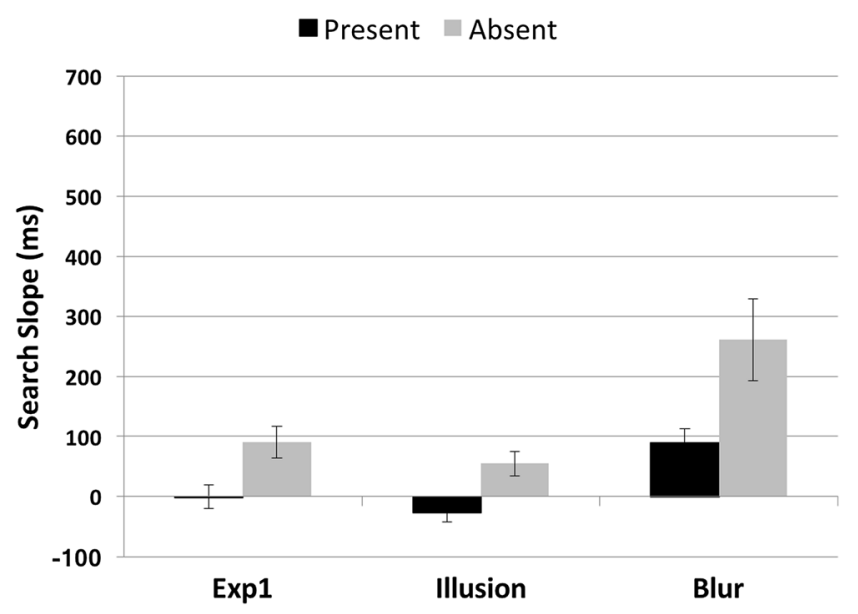

Fig. 10 Search slopes from Experiment 4. Summary of the target-present and target absent search slopes from the two conditions of Experiment 4. The corresponding slopes from Experiment 1 are also shown for comparison. See Table 1 for details of the RT $\times$ Set Size functions. Error bars show standard error of the mean 
motion. Our interest was whether search slopes would reflect this subjective loss of strength.

\section{Methods}

\section{Participants}

A new group of 12 participants (mean age $=26.8, \mathrm{SE}=2.2$; seven female; ten right-handed) were recruited from the University of Malta academic community. Group sample size was determined prior to data collection as described above. All participants had normal or corrected-to-normal vision and gave written informed consent before taking part in the experiment. They were naïve as to the purpose of the research and were not experienced psychophysical observers.

The study was conducted in accordance with the Ethics and Data Protection guidelines of the University of Malta and conformed to the Code of Ethics of the World Medical Association (Declaration of Helsinki).

\section{Stimuli}

In Experiment 4, stimuli were displayed at the center of the screen in an invisible $2 \times 2$ grid that subtended $23 \times 23^{\circ}$ visual angle. On a given trial, there could be two, three, or four items within the grid. The target and distractor items consisted of "quartets" made from four concentric rings. Figure 9 shows a display with a set size of four quartets. The target stimulus (lower right quartet) consisted of two ring groups having $\mathrm{CCW}$ rotation (upper left, lower right rings) and two having $\mathrm{CW}$ rotation (upper right, lower left rings). The opponent directions of neighboring rings is also a feature of the "Rotating Snakes" illusion, and here we use it to try and enhance the overall sense of illusory motion. The other three quartets in Fig. 5 are distractor items. Each ring was a control stimulus, with adjacent pairs being mirror reversed.

Each quartet subtended approximately $10.5^{\circ}$ visual angle and was separated from adjacent rings by $2.2^{\circ}$. Each set of rings within a quartet subtended $4.8^{\circ}$ and were separated from each other by approximately $0.7^{\circ}$ visual angle, the diameter of the atoms in the outer ring. The items in each subsequent ring were scaled down by $60 \%$, as described above. The screen background was set to a slightly lighter uniform gray (RGB values: 150) than in Experiment 1 (RGB values: 100) as the between-trials negative afterimage was stronger with the grid arrangement of stimuli.

Experiment 4 also contained a Blur control condition. We low-pass filtered the target and distractor images using a firstorder Butterworth filter, with a cut-off frequency of $20 \mathrm{~Hz}$. Figure 2 (lower panel) shows a single stimulus ring blurred with this method. It leaves the color differences and the overall layout of the stimuli almost completely intact, but substantially reduced the detail at the edges of the individual atomic elements. As expected, given that these differences are thought to drive the illusion, subjectively, the target stimulus now conveyed a weaker, although still present, sense of illusory motion. Note that in Experiment 4, blurring was applied to all elements in a quartet.

\section{Procedure}

In Experiment 4, we used an additional training step to introduce the two types of quartet that would be used in the visual search task. We did this to ensure that participants focused their search at the level of the quartet, not at the level of the individual rings. Prior to the main experiment, we presented target and distractor quartets from the illusion condition one at a time on the screen. Participants were asked to report, via a key press, whether they experienced illusory motion. They completed 60 trials of this $2 \mathrm{AFC}$ task.

Participants were then familiarized with the search task and were given practice before each experimental block. They then completed one block of Illusion trials and one block of Blur trials. We chose this fixed block order so that performance in the main illusion condition could serve as a clear baseline against which to judge the impact of blurring. Giving participants equal prior experience with the intact illusion would thus provide a more conservative estimate of the cost of blurring. Each block consisted of 120 trials using a 2 (present/absent $) \times 3($ set size $) \times 20$ (repetitions) design, presented in a different random order to each participant. The entire session took approximately 20 mins.

\section{Results}

Details of the RT $\times$ Set Size functions are given in Table 1 . Figure 10 summarizes the average search slopes for the two conditions of Experiment 4, in comparison to those seen in Experiment 1. As in Experiment 1, illusory motion gave rise to target-present search slopes that did not differ from zero, $\mathrm{t}(11)=-1.8, \mathrm{n} . \mathrm{s} ., \mathrm{d}=-0.5$. Search in the Blur condition, which subjectively gave rise to a weak sense of motion, had a slope that did differ from zero, $\mathrm{t}(11)=3.9, \mathrm{p}<0.01, \mathrm{~d}=1.1$. Although search was inefficient with blurred stimuli $(\mathrm{M}=$ $89 \mathrm{~ms} /$ item, $\mathrm{SE}=23$ ), the set size cost was much smaller than the slopes seen in Experiment 2 (see Fig. 6, Table 1).

To further explore the overall pattern of responses in Experiment 4, we used the same 2 (Condition) $\times 2$ (Target) $\times 3$ (Set Size) design as in Experiment 2. In Experiment 4, all factors were within subjects, leading to a fully repeated measures design. Also, as a Sphericity violation was detected for the main effect of Set Size, Greenhouse-Geisser corrections were applied to the appropriate degrees of freedom.

Responses in the Illusion condition $(\mathrm{M}=895 \mathrm{~ms}, \mathrm{SE}=55)$ were consistently faster than in the Blur condition $(\mathrm{M}=1,440$ $\mathrm{ms}, \mathrm{SE}=130$ ), giving rise to a main effect of Condition, 
$\mathrm{F}(1,11)=33.8, \mathrm{MSE}=315,665, \mathrm{p}<.001, \eta_{p}{ }^{2}=0.76$. Target present responses $(\mathrm{M}=1,017 \mathrm{~ms}, \mathrm{SE}=70)$ were consistently faster than target absent responses $(\mathrm{M}=1,317 \mathrm{~ms}, \mathrm{SE}=112)$, giving rise to a significant main effect of Target, $F(1,11)=$ 23.5, MSE $=138,345, \mathrm{p}<.01, \eta_{p}{ }^{2}=0.68$. The main effect of Set Size was also significant, $\mathrm{F}(1.15,12.6)=13.0$, MSE $=$ $58,115, \mathrm{p}<.01, \eta_{p}{ }^{2}=0.54$.

These main effects need to be interpreted within the context of significant two-way interactions. The Condition $\times$ Target interaction appears to be mostly driven by highly elevated responses in the Blur condition on Target Absent trials, $\mathrm{F}(1,11)=9.4, \mathrm{MSE}=82,659, \mathrm{p}<.05, \eta_{p}{ }^{2}=0.46$. The Condition $\times$ Set Size interaction arises as the responses rise more steeply in the more difficult Blur condition, $\mathrm{F}(2,22)=$ 16.8, MSE $=18,789, \mathrm{p}<.01, \eta_{p}{ }^{2}=0.6$. The Target $\times$ Set Size interaction simply reflects the more rapid rise in responses in the Target Absent trials, $\mathrm{F}(2,22)=10.6, \mathrm{MSE}=181,185$, $\mathrm{p}<$ $.01, \eta_{p}{ }^{2}=0.5$. The three-way interaction was not significant, $\mathrm{F}(2,22)=2.5, \mathrm{MSE}=20,277$, n.s., $\eta_{p}{ }^{2}=0.19$.

\section{Discussion}

The results of Experiment 4 replicate those of Experiments 1 and 3. Illusory motion, even in a more complex display, was able to automatically guide attention, leading to a flat search function. When the displays were modified so that the illusion was weakened, but still present, search was inefficient, albeit much faster than the control stimuli in Experiment 2. With reference to Table 2, we also note that the reduction in search efficiency was accompanied by an increase in error rates for the Blur condition. Such performance changes suggest that visual search could provide a useful tool in future studies to objectively measure the strength of illusory motion. It also suggests that weak illusory motion may not cross some quantitative threshold required in order to support efficient search. We return to this issue in the General discussion in relation to previous reports of individual differences in subjective awareness of illusory motion.

\section{General discussion}

In the current work, we have shown that the onset of illusory motion can pre-attentively guide perception in parallel across the visual field. This suggests that illusory motion, in addition to real-motion, can be treated as a basic visual feature (Treisman \& Gelade, 1980; Wolfe \& Horowitz, 2017). From previous neuroimaging (Ashida et al., 2012; Murakami et al., 2006; Zeki et al., 1993) and neurophysiological (Conway et al., 2005) studies it is known that viewing illusory motion leads to activation in early visual areas usually associated with real-motion processing. Our finding of "pop-out" provides clear behavioral evidence that such early activation may be sufficient for the detection of illusory motion, without the need to postulate the involvement of higher-level cognitive processing (Davis \& Driver, 1994; Zeki et al., 1993; see Kawahara et al., 1996 for similar search-based evidence in relation to illusory line motion).

As already noted, previous studies have found neural responses to illusory motion at many levels of the visual system (Ashida et al., 2012; Conway et al., 2005; Kuriki et al., 2008) and even beyond (Zeki et al., 1993). While the strong visual onsets in our displays would clearly activate early visual areas (V1), there are several reasons to believe that later and more specialized motion areas, specifically hMT+, are crucially involved (Born \& Bradley, 2005; Zeki et al., 1991). To begin with, the nature of our stimuli - with relatively large atomic elements compared to standard "Rotating Snakes"- clearly requires substantial spatial integration.

Furthermore, while target-present search slopes were flat, the intercepts of these functions were always well above $500 \mathrm{~ms}$ (see Tables 1 and 3), indicating that considerable temporal integration is also involved in the detection of illusory motion. Finally, a TMS study on the related "Enigma" illusion (Leviant, 1996) found that disruption of V1 had very little impact on subjective experience compared to stimulation of hMT+ (Ruzzoli et al., 2011). This latter finding, which we hope to verify with our own stimuli, raises the intriguing possibility that illusory motion might involve activation of hMT+ via retino-collicular rather than retino-thalamic pathways (Beltramo \& Scanziani, 2019). While we can only speculate, such a link might also help explain the very tight coupling between eye movements and illusory experience during free viewing. We return to the issue of eye movements shortly.

The main motivation for conducting Experiment 3, was to determine whether the relatively slow detection times observed in Experiment 1 were a specific characteristic of illusory motion processing. Clearly, they are not. Although we need to be cautious in interpreting the second main finding of Experiment 3 - that illusory motion targets were detected more quickly than speed-matched real motion targets - until it has been further verified, it certainly raises a number of interesting questions. Why would illusory motion be detected more quickly than real motion if the same neural mechanisms are responsible for both?

One possibility, already mentioned, is that illusory motion takes a different (or additional) route to reach motion sensitive regions. Another possibility is that additional factors act as a catalyst, speeding up the processing of illusory motion. For example, although Experiment 2 clearly showed that structural differences alone would not support efficient target detection, the integration of shape and motion signals in illusion targets (Fig. 2) could conceivably lead to a speed advantage, possibly by involving additional neural mechanisms, such as those in 
the superior temporal sulcus (STS; Shiffrar, 1994). In the real motion condition of Experiment 3, it was a structurally incoherent distractor item (Fig. 2) that was physically rotated.

Another potential catalyst could be eye movements. Throughout the history of illusory motion, there have been persistent suggestions that such phenomena originate in the eye rather than the brain (Beer et al., 2008; Gregory, 1993, 1994; Mon-Williams \& Wann, 1996; Murakami et al., 2006; Otero-Millan et al., 2012; Troncoso, Macknik, Otero-Millan, \& Martinez-Conde, 2008; Zanker, Doyle, \& Walker, 2003). Most recently, Martinez-Conde and colleagues (Otero-Millan et al., 2012; Troncoso et al., 2008) have shown convincingly that during prolonged viewing, fixational eye movements (FEMs) correlate strongly with the perceptual experience of illusory motion. Could FEMs contribute to the speeded detection of illusory motion observed in Experiment 3?

In order to approach this question, it is first important to note that the claim from previous studies is not that FEMs directly "cause" illusory motion, rather that they serve as a "trigger," giving rise to global visual transients that facilitate later cortical processing. As we did not measure eye movements in our studies, we can only speculate that they may have been able to exert some influence in the current tasks. One problem with this idea would be that our displays were relatively brief (most target-present responses occurred in less than a second) and the strong onset transients in our displays would probably have dominated the FEM transients, as this is known to be the case for up to $4 \mathrm{~s}$ after stimulus onset (OteroMillan et al., 2012).

Before leaving the topic of eye movements, we should also note that the relatively slow response rates in some conditions could also suggest the involvement of overt, saccadic eye movements. As already noted, it is almost certain that during the control conditions of Experiment 2, participants were required to fixate subsequent items in the absence of illusory motion. Again, as we did not use an eye tracker, we need to be cautious, but the results of Experiment 3 seem to argue against a crucial role for saccadic eye movements in the more efficient illusion conditions. That is, the four experienced observers were well practiced at maintaining fixation - as they were instructed to do - and their results were qualitatively the same as those of our naïve participants in Experiments 1 and 3. If nothing else, then, we feel confident in asserting that our main pattern of results does not require the generation of saccadic eye movements, even if we cannot rule out their occurrence with our naïve participants.

Finally, returning to the question of why the detection of illusory motion was speeded, it is possible that our nulling technique simply underestimated the matching point for real motion. A useful way forward might be to run a version of the search task where we parametrically vary real motion speed around the fairly consistent $2 \%$ s estimate for illusory motion.
At some point, faster responses should be observed for the real motion, and the change-over point may shed some additional light on the mechanisms involved.

Moving to another topic, Fraser and Wilcox (1979) reported that almost $25 \%$ of their participants did not subjectively experience illusory motion. A number of later studies also confirmed some level of perceptual dimorphism (Billino et al., 2009; Gori, Mascheretti, et al., 2015; Naor-Raz \& Sekuler, 2000). Interestingly, we found no evidence of such individual differences in the current work. All participants subjectively reported clear impressions of illusory motion in the relevant conditions, and the overall search data would certainly seem to back this up. Furthermore, we informally presented our main stimuli to 450 visitors at a science fair in Belgrade, Serbia. Only 12 individuals (seven adults/five children) failed to localize and describe the illusory motion. It would seem that the "optimized" nature of Kitaoka's displays - features that we adopted for our stimuli - provide strong enough stimulation to bring the illusory motion subjectively above threshold for almost all observers.

A less well-reported aspect of the Fraser and Wilcox (1979) individual differences data is their finding that the performance of participants who had no subjective awareness of the illusion still showed clear modulation when asked to null physically rotating rings that were paired with (invisible) illusory motion. This suggests that weaker signals were present for these observers, but simply failed to cross the threshold of awareness. This hints at a quantitative rather than a qualitative difference in detection ability between those who experience the illusion and those who do not. Less efficient search with the experimentally weakened illusory motion of the Blur condition in Experiment 4 also supports this idea.

Finally, we hope that over and above our demonstration of parallel processing, the current work makes a useful contribution by reemphasizing that visual search can be a flexible objective measure in the context of illusions (Davis \& Driver, 1994; Enns \& Rensink, 1990; Kawahara et al., 1996; Li, Cave, \& Wolfe, 2008; Treisman \& Gelade, 1980). In future, rather than simply asking whether illusory motion is experienced or to rate its subjective strength, it should be possible to also gauge objective strength by examining search efficiency.

Acknowledgements We would like to thank Akiyoshi Kitaoka for permission to reproduce his beautiful illusions. We would also like to thank Patrick Cavanagh, Todd Horowitz, Árni Kristjánsson, Shin'ya Nishida, Quoc Vuong, and Ron Rensink for commenting on earlier drafts of this manuscript and for providing usual comments. Ivana Tomić provided invaluable assistance in obtaining the luminance and colorimetry readings provided in Table S1. We are also grateful to Marthese Borg, Becky Stivala, Matthew Fenech, and Nehemie Bikin-kita for help with data collection and to Dejan Todorović and Quoc Vuong for participating in Experiment 3. This research was supported in part by the Ministry of Education and Science of the Republic of Serbia, Grants No. 179033 and III 47020 . 
Author Contributions IMT and SZ contributed equally to the design, analysis, and writing of this paper.

\section{References}

Abrams, R. A., \& Christ, S. E. (2003). Motion onset captures attention. Psychological Science, 14(5), 427-432. https://doi.org/10.1111/ 1467-9280.01458

Ashida, H., Kuriki, I., Murakami, I., Hisakata, R., \& Kitaoka, A. (2012). Direction-specific fMRI adaptation reveals the visual cortical network underlying the "rotating snakes" illusion. NeuroImage, 61(4), 1143-1152. https://doi.org/10.1016/j.neuroimage.2012.03.033

Ashida, H., Lingnau, A., Wall, M. B., \& Smith, A. T. (2007). fMRI adaptation reveals separate mechanisms for first-order and secondorder motion. Journal of Neurophysiology, 97(2), 1319-1325. https://doi.org/10.1152/jn.00723.2006

Ashida, H., Seiffert, A. E., \& Osaka, N. (2001). Inefficient visual search for second-order motion. Journal of the Optical Society of America A, 18(9), 2255. https://doi.org/10.1364/JOSAA.18.002255

Backus, B. T., \& Oruç, I. (2005). Illusory motion from change over time in the response to contrast and luminance. Journal of Vision, 5(11), 10. https://doi.org/10.1167/5.11.10

Beer, A. L., Heckel, A. H., \& Greenlee, M. W. (2008). A motion illusion reveals mechanisms of perceptual stabilization. PLoS ONE, 3(7), e2741. https://doi.org/10.1371/journal.pone.0002741

Beltramo, R., \& Scanziani, M. (2019). A collicular visual cortex: Neocortical space for an ancient midbrain visual structure. Science, 363(6422), 64-69. https://doi.org/10.1126/science.aau7052

Billino, J., Hamburger, K., \& Gegenfurtner, K. R. (2009). Age effects on the perception of motion illusions. Perception, 38(4), 508-521. https://doi.org/10.1068/p5886

Born, R. T., \& Bradley, D. C. (2005). Structure and function of visual area MT. Annual Review of Neuroscience, 28(1), 157-189. https://doi. org/10.1146/annurev.neuro.26.041002.131052

Brainard, D. H. (1997). The Psychophysics Toolbox. Spatial Vision, 10, 433-436.

Bülthoff, H., \& Götz, K. G. (1979). Analogous motion illusion in man and fly. Nature, 278(5705), 636-638. https://doi.org/10.1038/ $278636 \mathrm{a} 0$

Cavanagh, P., Labianca, A. T., \& Thornton, I. M. (2001). Attention-based visual routines: Sprites. Cognition, 80(1-2), 47-60.

Chun, M. M., \& Wolfe, J. M. (1996). Just say no: How are visual searches terminated when there is no target present? Cognitive Psychology, 30(1), 39-78. https://doi.org/10.1006/cogp.1996.0002

Conway, B. R., Kitaoka, A., Yazdanbakhsh, A., Pack, C. C., \& Livingstone, M. S. (2005). Neural basis for a powerful static motion illusion. The Journal of Neuroscience: The Official Journal of the Society for Neuroscience, 25(23), 5651-5656. https://doi.org/10. 1523/JNEUROSCI.1084-05.2005

Davis, G., \& Driver, J. (1994). Parallel detection of Kanizsa subjective figures in the human visual system. Nature, 371(6500), 791-793. https://doi.org/10.1038/371791a0

Dick, M., Ullman, S., \& Sagi, D. (1987). Parallel and serial processes in motion detection. Science (New York, N.Y.), 237(4813), 400-402.

Eagleman, D. M. (2001). TIMELINE: Visual illusions and neurobiology. Nature Reviews Neuroscience, 2(12), 920-926. https://doi.org/10. $1038 / 35104092$

Enns, J. T., \& Rensink, R. A. (1990). Sensitivity to three-dimensional orientation in visual search. Psychological Science, 1(5), 323-326. https://doi.org/10.1111/j.1467-9280.1990.tb00227.x

Faubert, J., \& Herbert, A. M. (1999). The peripheral drift illusion: A motion illusion in the visual periphery. Perception, 28(5), 617-621.

Fraser, A., \& Wilcox, K. J. (1979). Perception of illusory movement. Nature, 281(5732), 565-566. https://doi.org/10.1038/281565a0
Gori, S., Agrillo, C., Dadda, M., \& Bisazza, A. (2015). Do fish perceive illusory motion? Scientific Reports, 4(1). https://doi.org/10.1038/ srep06443

Gori, S., \& Hamburger, K. (2006). A new motion illusion: The rotatingtilted-lines illusion. Perception, 35(6), 853-857. https://doi.org/10. $1068 / \mathrm{p} 5531$

Gori, S., Mascheretti, S., Giora, E., Ronconi, L., Ruffino, M., Quadrelli, E., ... Marino, C. (2015). The DCDC2 Intron 2 deletion impairs illusory motion perception unveiling the selective role of magnocellular-dorsal stream in reading (dis)ability. Cerebral Cortex, 25(6), 1685-1695. https://doi.org/10.1093/cercor/bhu234

Gregory, R. L. (1968). Visual illusions. Scientific American, 219(5), 66-76.

Gregory, R. L. (1993). A comment: Mackay rays shimmer due to accommodation changes. Proceedings of the Royal Society B: Biological Sciences, 253(1336), 123-123. https://doi.org/10.1098/rspb.1993. 0090

Gregory, R. L. (1994). Under the carpet. Perception, 23(7), 741-744. https://doi.org/10.1068/p230741

Hisakata, R., \& Murakami, I. (2008). The effects of eccentricity and retinal illuminance on the illusory motion seen in a stationary luminance gradient. Vision Research, 48(19), 1940-1948. https://doi.org/ 10.1016/j.visres.2008.06.015

Horowitz, T. S., Wolfe, J. M., DiMase, J. S., \& Klieger, S. B. (2007). Visual search for type of motion is based on simple motion primitives. Perception, 36(11), 1624-1634. https://doi.org/10.1068/ p5683

Kawahara, J. 'ichiro, Yokosawa, K., Nishida, S. 'ya, \& Sato, T. (1996). Illusory line motion in visual search: Attentional facilitation or apparent motion? Perception, 25(8), 901-920. https://doi.org/10.1068/ p250901

Kitaoka, A., \& Ashida, H. (2003). Phenomenal characteristics of the peripheral drift illusion. Vision, 15, 261-262.

Kleiner, M., Brainard, D. H., Pelli, D. G., Ingling, A., Murray, R., Broussard, C., et al. (2007). What's new in Psychtoolbox-3. Perception, 36(14), 1.

Kuriki, I., Ashida, H., Murakami, I., \& Kitaoka, A. (2008). Functional brain imaging of the rotating snakes illusion by fMRI. Journal of Vision, 8(10), 16-16. https://doi.org/10.1167/8.10.16

Léviant, I. (1982). Illusory motion within still pictures: The L-effect. Leonardo, 15(3), 222-223.

Leviant, I. (1996). Does "brain-power" make enigma spin? Proceedings of the Royal Society B: Biological Sciences, 263(1373), 997-1001. https://doi.org/10.1098/rspb.1996.0147

Li, X., Cave, K. R., \& Wolfe, J. M. (2008). Kanizsa-type subjective contours do not guide attentional deployment in visual search but line termination contours do. Perception \& Psychophysics, 70(3), 477-488. https://doi.org/10.3758/PP.70.3.477

Mackay, D. M. (1957). Moving visual images produced by regular stationary patterns. Nature, 180(4591), 849-850. https://doi.org/10. 1038/180849a0

Mayer, K. M., Vuong, Q. C., \& Thornton, I. M. (2015). Do people "Pop Out"? PLoS ONE, 10(10), e0139618. https://doi.org/10.1371/ journal.pone.0139618

Mayer, K. M., Vuong, Q. C., \& Thornton, I. M. (2017). Humans are detected more efficiently than machines in the context of natural scenes: Humans are detected more efficiently than machines. Japanese Psychological Research, 59(2), 178-187. https://doi.org/ 10.1111/jpr.12145

Mon-Williams, M., \& Wann, J. P. (1996). An illusion that avoids focus. Proceedings of the Royal Society B: Biological Sciences, 263(1370), 573-578. https://doi.org/10.1098/rspb.1996.0086

Murakami, I., Kitaoka, A., \& Ashida, H. (2006). A positive correlation between fixation instability and the strength of illusory motion in a static display. Vision Research, 46(15), 2421-2431. https://doi.org/ 10.1016/j.visres.2006.01.030 
Nakayama, K., \& Silverman, G. H. (1986). Serial and parallel processing of visual feature conjunctions. Nature, 320(6059), 264-265. https:// doi.org/10.1038/320264a0

Naor-Raz, G., \& Sekuler, R. (2000). Perceptual dimorphism in visual motion from stationary patterns. Perception, 29(3), 325-335.

Nishida, S. 'ya, Sasaki, Y., Murakami, I., Watanabe, T., \& Tootell, R. B. H. (2003). Neuroimaging of direction-selective mechanisms for second-order motion. Journal of Neurophysiology, 90(5), 32423254. https://doi.org/10.1152/jn.00693.2003

Normand, M. P. (2016). Less is more: Psychologists can learn more by studying fewer people. Frontiers in Psychology, 7. https://oi.org/ 10.3389/fpsyg.2016.00934

Otero-Millan, J., Macknik, S. L., \& Martinez-Conde, S. (2012). Microsaccades and blinks trigger illusory rotation in the "rotating snakes" illusion. Journal of Neuroscience, 32(17), 6043-6051. https://doi.org/10.1523/JNEUROSCI.5823-11.2012

Pelli, D. G. (1997). The VideoToolbox software for visual psychophysics: Transforming numbers into movies. Spatial Vision, 10(4), 437-442.

Pinna, B., \& Brelstaff, G. J. (2000). A new visual illusion of relative motion. Vision Research, 40(16), 2091-2096. https://doi.org/10. 1016/S0042-6989(00)00072-9

Prins, N., \& Kingdom, F. A. A. (2018). Applying the model-comparison approach to test specific research hypotheses in psychophysical research using the Palamedes Toolbox. Frontiers in Psychology, 9. https://doi.org/10.3389/fpsyg.2018.01250

Rensink, R. A. (2000). Visual search for change: A probe into the nature of attentional processing. Visual Cognition, 7(1-3), 345-376. https://doi.org/10.1080/135062800394847

Rensink, R. A., O’Regan, J. K., \& Clark, J. J. (1997). To see or not to see: The need for attention to perceive changes in scenes. Psychological Science, 8(5), 368-373. https://doi.org/10.1111/j.1467-9280.1997. tb00427.x

Rousselet, G. A., \& Wilcox, R. R. (2018). Reaction times and other skewed distributions: Problems with the mean and the median. https://doi.org/10.1101/383935

Ruzzoli, M., Gori, S., Pavan, A., Pirulli, C., Marzi, C. A., \& Miniussi, C. (2011). The neural basis of the Enigma illusion: A transcranial magnetic stimulation study. Neuropsychologia, 49(13), 3648-3655. https://doi.org/10.1016/j.neuropsychologia.2011.09.020

Seiffert, A. E. (2003). Functional MRI studies of human visual motion perception: Texture, luminance, attention and after-effects. Cerebral Cortex, 13(4), 340-349. https://doi.org/10.1093/cercor/13.4.340

Shapiro, A., \& Todorović, D. (Eds.). (2016). The Oxford compendium of visual illusions. Oxford: Oxford University Press.

Shiffrar, M. (1994). When what meets where. Current Directions in Psychological Science, 3(3), 96-101. https://doi.org/10.1111/14678721.ep10770450
Smith, A. T., Greenlee, M. W., Singh, K. D., Kraemer, F. M., \& Hennig, J. (1998). The processing of first- and second-order motion in human visual cortex assessed by functional magnetic resonance imaging (fMRI). The Journal of Neuroscience, 18(10), 3816-3830. https:// doi.org/10.1523/JNEUROSCI.18-10-03816.1998

Smith, P. L., \& Little, D. R. (2018). Small is beautiful: In defense of the small-N design. Psychonomic Bulletin \& Review, 25(6), 2083-2101. https://doi.org/10.3758/s13423-018-1451-8

Treisman, A. M., \& Gelade, G. (1980). A feature-integration theory of attention. Cognitive Psychology, 12(1), 97-136.

Troncoso, X. G., Macknik, S. L., Otero-Millan, J., \& Martinez-Conde, S. (2008). Microsaccades drive illusory motion in the Enigma illusion. Proceedings of the National Academy of Sciences, 105(41), 1603316038. https://doi.org/10.1073/pnas.0709389105

Wichmann, F. A., \& Hill, N. J. (2001). The psychometric function: II Bootstrap-based confidence intervals and sampling. Perception \& Psychophysics, 63(8), 1314-1329. https://doi.org/10.3758/ BF03194545

Wolfe, J. M. (2010). Visual search. Current Biology, 20(8), R346-R349. https://doi.org/10.1016/j.cub.2010.02.016

Wolfe, J. M., \& Horowitz, T. S. (2004). Opinion: What attributes guide the deployment of visual attention and how do they do it? Nature Reviews Neuroscience, 5(6), 495-501. https://doi.org/10.1038/ nrn1411

Wolfe, J. M., \& Horowitz, T. S. (2017). Five factors that guide attention in visual search. Nature Human Behaviour, 1(3), 58. https://doi.org/10. 1038/s41562-017-0058

Wolfe, J. M., \& Utochkin, I. S. (2019). What is a preattentive feature? Current Opinion in Psychology, 29, 19-26. https://doi.org/10.1016/ j.copsyc.2018.11.005

Zanker, J. M., Doyle, M., \& Walker, R. (2003). Gaze stability of observers watching op art pictures. Perception, 32(9), 1037-1049. https://doi.org/10.1068/p5128

Zeki, S., Watson, J. D., Lueck, C. J., Friston, K. J., Kennard, C., \& Frackowiak, R. S. (1991). A direct demonstration of functional specialization in human visual cortex. The Journal of Neuroscience: The Official Journal of the Society for Neuroscience, 11(3), 641-649.

Zeki, S., Watson, J. D. G., \& Frackowiak, R. S. J. (1993). Going beyond the information given: The relation of illusory visual motion to brain activity. Proceedings of the Royal Society B: Biological Sciences, 252(1335), 215-222. https://doi.org/10.1098/rspb.1993.0068

Publisher's note Springer Nature remains neutral with regard to jurisdictional claims in published maps and institutional affiliations. 